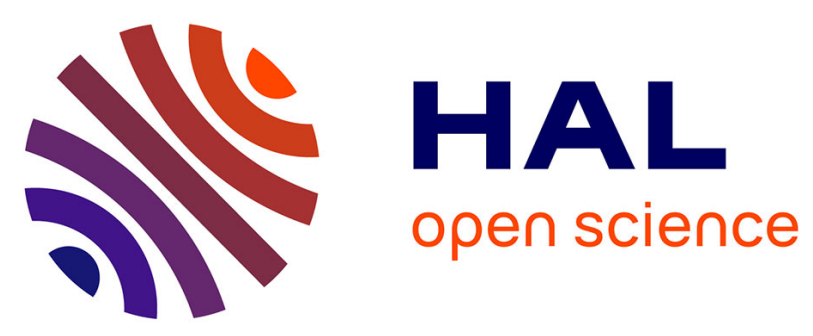

\title{
Self-consistent modelling of heating synergy between NBI and ICRH in JET deuterium plasmas
}

J. Joly, J. Garcia, Frédéric Imbeaux, R. Dumont, M. Schneider, T. Johnson, Jean-François Artaud

\section{> To cite this version:}

J. Joly, J. Garcia, Frédéric Imbeaux, R. Dumont, M. Schneider, et al.. Self-consistent modelling of heating synergy between NBI and ICRH in JET deuterium plasmas. Plasma Physics and Controlled Fusion, 2019, 61, pp.075017. 10.1088/1361-6587/ab1f54 . cea-02196581

\section{HAL Id: cea-02196581 https://hal-cea.archives-ouvertes.fr/cea-02196581}

Submitted on 29 Jul 2019

HAL is a multi-disciplinary open access archive for the deposit and dissemination of scientific research documents, whether they are published or not. The documents may come from teaching and research institutions in France or abroad, or from public or private research centers.
L'archive ouverte pluridisciplinaire HAL, est destinée au dépôt et à la diffusion de documents scientifiques de niveau recherche, publiés ou non, émanant des établissements d'enseignement et de recherche français ou étrangers, des laboratoires publics ou privés. 


\title{
Self-consistent modelling of heating synergy between NBI and ICRH in JET deuterium plasmas
}

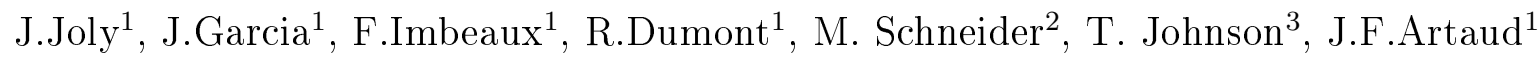 \\ January 22, 2019
}

${ }^{1}$ CEA, IRFM, F-13108 Saint-Paul-lez-Durance, France

${ }^{2}$ ITER Organization, Route de Vinon-sur-Verdon, CS 90 046, 13067 St. Paul-lez-Durance, France 2ITER Organization, Route de Vinon-sur-Verdon, CS 90 046, 13067 St. Paul-lez-Durance, France

${ }^{3}$ KTH Royal Institute of Technology, 10044 Stockholm, Sweden

\begin{abstract}
Auxiliary heating is essential to initiate fusion in future tokamaks. In particular, ion heating tends to maximize the alpha power generation by increasing the thermal ion temperature. In order to simulate the plasma heating by Ion Cyclotron Radio Frequency waves (ICRF), the EVE code, a full-wave code for IC wave propagation, and SPOT, an orbit following Monte-Carlo code combined with the RFOF library which calculates the absorption of wave by ions, have been coupled together. This new package is used for simulating JET plasmas with strong interplay between ICRH and NBI (Neutral Beam Injection). Simulations shows that up to $20 \%$ of the neutron rate generated in recent JET $\mathrm{D}$ plasmas is due to the synergy between both heating mechanisms. However, the $\mathrm{H}$ concentration plays a critical role on such interplay, as beyond $2 \%$, the synergy efficiency weakens. Therefore, the control of the $\mathrm{H}$ concentration is mandatory for optimizing the fusion reaction rate generation at JET.
\end{abstract}

\section{Introduction}

The ultimate goal of fusion plasmas is to generate clean and efficient energy. For that purpose, it is mandatory to heat the plasma, mainly the ions, in order to generate enough fusion power from the DT reactions. Among the several heating mechanisms proposed for plasma heating, Ion Cyclotron Ressonant Heating (ICRH) and Neutral Beam Injection (NBI) have been demonstrated to efficiently heat the ions $[1,2,3,4,5]$. At least at JET, such combination of heating mechanisms is being used to prepare the future operation in DT by maximizing the fusion power generation obtained in D plasmas as a first step for boosting the alpha power generation in DT plasmas [6]. A significant improvement in fusion reaction, that results in an increase of neutron rate, has been obtained in recent campaigns by increasing the ICRH and NBI power used [7].

A key point for burning plasmas, in particular for ITER, is the proper calculation and predictability of neutron rates as the activation of the different wall components will critically depend on them. Therefore, the analysis of such predictability has been performed in JET plasmas heated by ICRH and NBI. In many cases the calculated neutron rates are too low just assuming the ion distribution function obtained from orbit following Monte-Carlo codes, such as NUBEAM, applied to the NBI heating $[8,9,10]$. A possible explanation is the interplay between the ICRH and the NBI heating. The acceleration of beam ions by the ICRF waves modifies the $\mathrm{D}$ distribution and consequently the neutron rate. For instance, the most common ICRH scenario at JET is the minority heating of $\mathrm{H}$ whose resonance layer also corresponds to the heating of the second harmonic of D. Therefore, the synergy between NBI heating, which inject D beams, and the second harmonic ICRH heating of D could achieve higher fusion reaction rate than single minority heating because of the extra energetic ions obtained.

The analysis of such physics with first principle codes is essential in order to further improve the neutron rate calculation. Additionally, the calculation of a self-consistent ion distribution function is essential as turbulence can be significantly reduced in the presence of energetic ions $[11,12,13]$. Finally, the extrapolation of D plasmas neutron rate to DT plasmas requires a correct understanding of the underlying physics as optimized ICRH heating schemes and cross sections significantly change from D to DT plasmas. 
Previous analyses of ICRH and NBI synergies have been performed with the PION code $[14,15,16]$ and SELFO $[17,18]$, but both codes have shortcomings. The PION code is based on significantly simplified models both for the wave propagation and the Fokker-Planck modelling of the distribution functions. The SELFO code, on the other hand, assumes a simplified geometry in its Fokker-Planck treatment of the resonating ion species.

In the present work, in order to increase the accuracy of the fast ion distribution modelling, the wave propagation and both its dependence and effect on the fast ion distribution are modelled self-consistently with respectively a $2 \mathrm{D}$ full-wave code [19] (EVE) and a Monte-Carlo orbit following 3D code [20] (SPOT/RFOF). These codes have been coupled using the frameworkof the European Integrated Modelling (ITM) [21], which has provided the background quantities required to perform the analyses, such as equilibrium and input temperature and density profiles.

The paper is divided as follows. In section 2, we describe the self-consistent coupling of the EVE / SPOT codes. In section 3, we present main parameters of JET hybrid discharge and input used. Section 4 presents the different results of the synergy modelling of NBI and ICRH, in particular we see in section (4.1) the results of NBI source modelling, in (4.2) the results of self-consistent simulations of NBI and ICRH heating synergy and in section (4.3) we compare NBI heating only with NBI + ICRH heating for neutron production. In section 5, we study the impact of minority species concentration on NBI and ICRH heating synergy.

\section{Self-consistent coupling of full wave code and Fokker-Plank code}

Modelling of fast ions accelerated by ICRH requires to solve Maxwell's equations to characterize the electric field of the wave in the plasma (eq.1) and to solve the Fokker-Planck equation to characterize the evolution of the ion distribution interacting with ICRF wave (eq.2).

$$
\begin{gathered}
\nabla \times \nabla \times \vec{E}=\frac{\omega^{2}}{c^{2}} \overleftrightarrow{\epsilon}\left(f_{i}\right) \cdot \vec{E}+i \omega \mu_{0} \dot{j}_{\text {ext }} \\
\frac{d f_{i}}{d t}=C\left(f_{i}\right)+Q\left(f_{i}, \vec{E}\right)+S_{N B I}
\end{gathered}
$$

where $\vec{E}$ is wave electric field, $\omega$ is the ICRF wave frequency, $c$ is speed of light, $\overleftrightarrow{\epsilon}$ is dielectric tensor, $\mu_{0}$ is magnetic permeability of vacuum, $\overrightarrow{j_{\text {ext }}}$ is current density of antenna, $f_{i}$ is distribution of resonant ion, $C\left(f_{i}\right)$ is collision operator and $Q\left(f_{i}, \vec{E}\right)$ is the quasi-linear operator of wave/particle interaction. To have a complete modelling, it is needed to take account feedback of distribution on field and inversely which means solving the two previous equations self-consistently.

To solve the ICRF wave propagation (cf eq.1) we used the EVE code which is described in [19]. This full wave code calculates the wave electric field by solving Maxwell's equations, using a variational principe. The quadratic dependence of the functional on the interaction Hamiltonian makes of EVE a second-order Finite Larmor Radius (FLR) code. The system of coordinates is $(s, \theta, \varphi)$, with s the radial coordinate, related to the poloidal flux by the relation $\nabla \Psi \equiv f(s) \nabla s$, and varying between 0 (centre) and 1 (edge) in the plasma volume. $\theta$ and $\varphi$ are the poloidal and toroidal angles. The warm dielectric tensor in equation 1 is calculated assuming the heated distribution function is given by a bi-Maxwellian, i.e.

$$
f\left(v_{\|}, v_{\perp}\right)=n_{f}\left(\frac{m_{f}}{2 \pi T_{\|}}\right)^{1 / 2} \exp \left(-\frac{m_{f} v_{\|}^{2}}{2 T_{\|}}\right)\left(\frac{m_{f}}{2 \pi T_{\perp}}\right) \exp \left(-\frac{m_{f} v_{\perp}^{2}}{2 T_{\perp}}\right)
$$

with $n_{f}$ fast ion density and $m_{f}$ its mass, $T_{\perp}, T_{\|}, v_{\perp}, v_{\|}$effective temperatures and velocity in perpendicular and parallel direction.

To solve the evolution of ion distribution (cf eq.2), we used SPOT code (Simulation of Particle Orbits in a Tokamak) [22], which is an orbit following Monte-Carlo code solving the Fokker-Planck's equation in five dimensions $(R, Z, \varphi, v, \chi)$, where $R, Z$ is the position of particle, $\varphi$ is a toroidal angle, $v$ is the particle velocity and $\chi=v_{\|} / v$ is the pitch angle. This code use Monte-Carlo operators to take account of collisions between each species of plasma [23] (i.e $C\left(f_{i}\right)$ in eq.2). The output of the code is a set of particles (markers) with different weights representing a sample of the fast deuterium population. To describe this sample as a bi-Maxwellian distribution, the parameters of the bi-Maxwellian are calculated from the markers as follows:

$$
\begin{cases}n_{\text {fast }}=\sum_{N} p_{N} & \\ W_{\|}=\frac{1}{2} m \sum_{N} v_{\| N}^{2} p_{N} & \rightarrow T_{\|}=2 \frac{W_{\|}}{q_{e} n_{\text {fast }}} \\ W_{\perp}=\frac{1}{2} m \sum_{N} v_{\perp N}^{2} p_{N} & \rightarrow T_{\perp}=\frac{W_{\perp}}{q_{e} n_{\text {fast }}}\end{cases}
$$


with $N$ is the number of markers, $p_{N}$ is the weight associated with the marker per unit volume and $W_{\perp, \|}$ is the perpendicular and parallel energy contents, so that the distribution is consistent with the calculation of the dielectric tensor.

To model the fast ion source created by NBI heating, we used NEMO code (NEutral beam MOdelling) detailed in [24] which compute the neutral beam ionization during neutral beam injection (NBI) in tokamak plasmas (i.e $S_{n b i}$ in eq.2), whose outputs can be used by a Monte-Carlo code like SPOT.

To model wave/particle interaction (i.e $Q\left(f_{i}, \vec{E}\right)$ in eq.2). , we used the RFOF library [25]. This code calculates the quasi-linear diffusion coefficient $D_{R F}[26]$ :

$$
D_{R F} \propto\left|E_{+} J_{n-1}\left(\frac{k_{\perp} v_{\perp}}{\omega_{c i}}\right)+E_{-} J_{n+1}\left(\frac{k_{\perp} v_{\perp}}{\omega_{c i}}\right)\right|^{2}
$$

where $\omega_{c i}$ is the ion cyclotron frequency, $E_{+}$and $E_{-}$are respectively the left-handed and right-handed electric field, $n$ is the harmonic index of the cyclotron resonance and $J_{n}$ are Bessel's functions. Kicks in the phase space variables are described by Monte Carlo operators constructed from the quasilinear coefficient $D_{R F}$ for each individual resonance point (where $\omega-n \omega_{c i}-k_{\|} v_{\|}-k_{\perp} V_{D}=0$ ).

The orbit following code requires long computation times. In order to keep it tractable, the ion distribution has been separated in two distributions, representing respectively the thermal and superthermal parts of the distribution. For this splitting we used a criteria on the deuterium temperature which is the following : if the temperature is greater than 1.5 times the thermal temperarture, the deuterium is considered as fast (i.e $T_{f a s t}>1.5 T_{i}$ ) [27]. This separation constitutes a reasonable approximation because we want to model the dynamics of fast ions created by NBI and accelerated by ICRH. Thus the number of particles required to represent the physical evolution of the distribution of the fast ions remains compatible with a tractable calculation time.

To do a self-consistent simulation of the NBI and ICRH heating synergy, we created a workflow combining NEMO / EVE / SPOT / RFOF, which is represented by figure 1. Firstly, we model the NBI source with NEMO/SPOT and the propagation of ICRF wave, with EVE, in the plasma taking into account the NBI source, which is represented by the blue box in figure 1. This step corresponds to a modelling without iteration between EVE/SPOT (i.e it $=0$ ). Secondly, we make several iterations between EVE/SPOT, which is represented by the red box in figure 1, to take into account feedback from the evolution of the distribution function on ICRH power. We thus account simultaneously for 1) the evolution of the fast D distribution function over time, as a result of the ICRH application on top of the NBI and 2) the self-consistent modification of the RF wave field and absorption properties. To determine whether the latter is important or not for this particular JET case, a simulation without self-consistent iterations between EVE and SPOT has been carried out and will be compared to the fully self-consistent one in section 4.2 .

SPOT has been extended by including the calculation of the neutron rate $R_{i j}$ (where $\mathrm{i}$ and $\mathrm{j}$ represent ionic species) for D-D, D-T and T-T fusion reactions as follows :

$$
R_{i j}=\frac{1}{\delta_{i j}} n_{i} n_{j} \cdot\langle\sigma v\rangle_{i j}
$$

with $n_{i, j}$ ion density and $\langle\sigma v\rangle_{i j}$ reaction rate between $\mathrm{i}$ and $\mathrm{j}$ species. For thermal interaction, the reaction rate is calculated with the equation 6 of reference [28]. For the beam-target interaction, the calculation of reaction rate is described in [29]. We have neglected the beam-beam interaction because the beam density is of the order of only a few percent. The neutron rate offers a way to benchmark the simulation results with experimental measurements. 


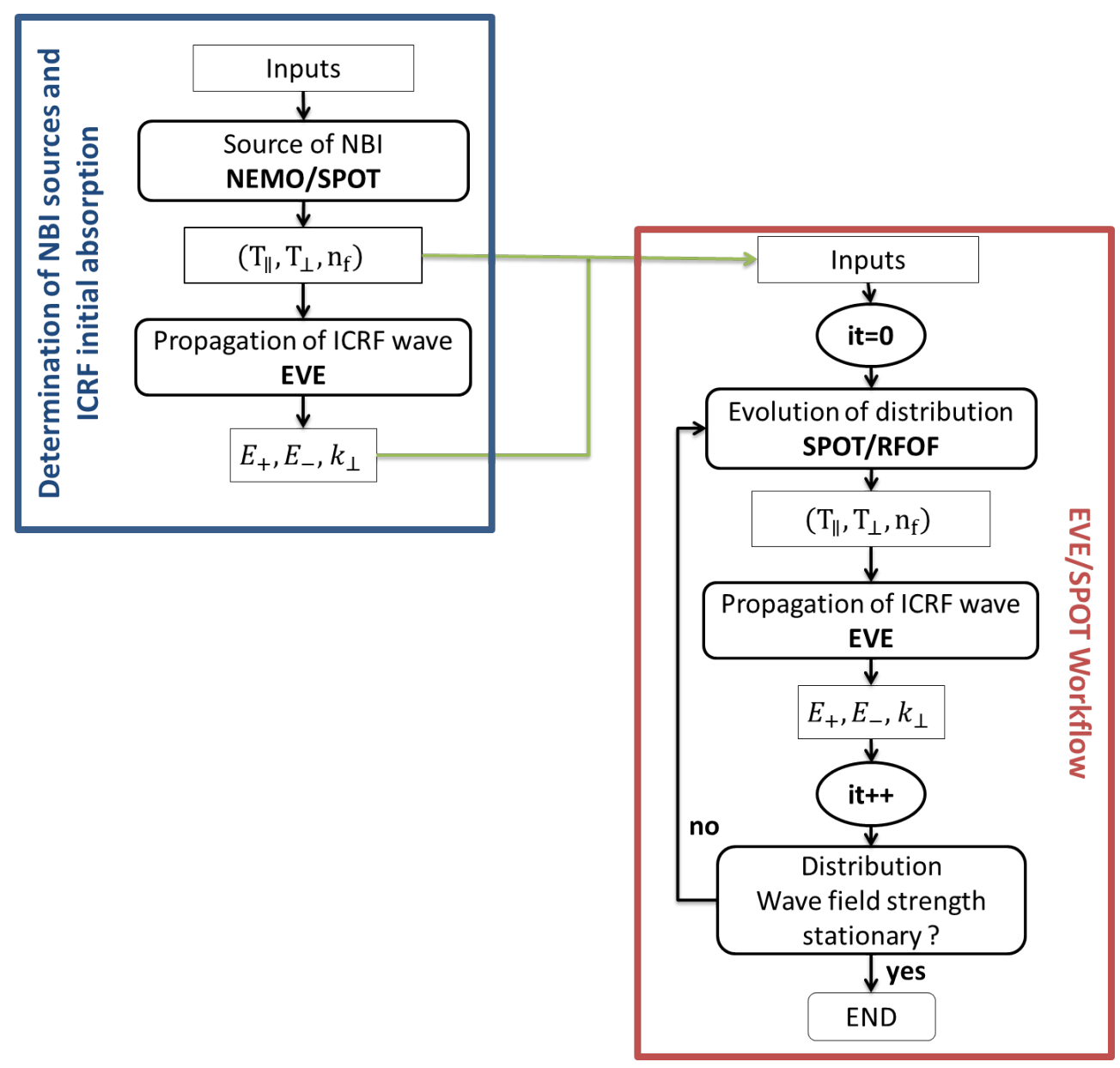

Figure 1: Schema of the self-consistent coupling workflow between EVE and SPOT

\section{Description of JET shot \#86614}

During the 2014 JET experimental campaign with the ITER-like-wall (ILW) and deuterium as the main gas, highperformance hybrid discharges have been achieved with combined deuterium neutral beam injection (NBI) and ion cyclotron resonance heating (ICRH). The heating power used in this discharge was 22MW of NBI with 5MW of ICRH. The JET hybrid discharges 86614 had the record of neutron rate $\left(R_{D D}=2.4 * 10^{16} s^{-1}\right)$ [30]. The main parameters for this discharge are summarized in table 1 at time 48s, corresponding to a stationary phase with maximum NBI and ICRH heating. The ICRH scenario used is the fundamental cyclotron frequency of minority hydrogen ions which coincides with the second harmonic cyclotron frequency of deuterium ions in the plasma center with a frequency of $\omega=42.5 \mathrm{MHz}$ $\left(\omega \approx \omega_{c H}=2 \omega_{c D}\right)$. The resonance layer of ICRH scenario is represented on figure 2 for all ion species entering in the plasma composition in the poloidal cros section. A previous analysis of this discharge has been carried out in reference [31], estimating that this discharge had an $\mathrm{H}$ concentration of about $2 \%$. 


\begin{tabular}{|l|l|}
\hline Parameters at $t=48 s$ & \\
\hline Major radius $R_{0}$ and minor radius $r_{a}(\mathrm{~m})$ & $2.89,0.94$ \\
Central magnetic field $B_{0}(T)$ & 2.9 \\
Plasma current $I_{p}(M A)$ & 2.5 \\
Normalized beta $\beta_{N}$ & 2.3 \\
$H_{98}$ & 1.10 \\
$q_{95}$ & 3.6 \\
Volume averaged and central electron temperature $\left\langle T_{e}\right\rangle, T_{e 0}(\mathrm{keV})$ & $2.8,7.7$ \\
Volume averaged and central electron density $\left\langle n_{e}\right\rangle, n_{e 0}\left(10^{19} \mathrm{~m}^{-3}\right)$ & $4.3,7.1$ \\
Concentration of $n_{H} / n_{e}, n_{B e} / n_{e}, n_{W} / n_{e}(\%)$ & $1.95,1.32,0.01$ \\
Neutron Rate of D-D reaction $R_{D D}\left(10^{16} s^{-1}\right)$ & 2.0 \\
\hline
\end{tabular}

Table 1: Main characteristics of the discharge 86614 at $48 \mathrm{~s}$

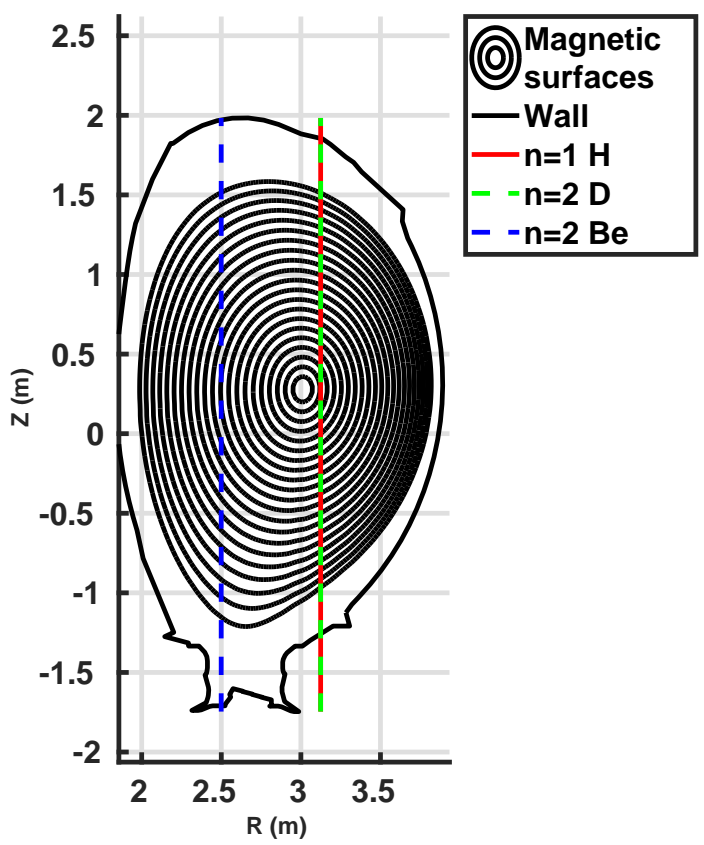

Figure 2: View in a poloidal cross-section of JET wall, magnetic surfaces from METIS, equilibrium and resonance layer of ICRH for antenna frequency $\omega=42.5 \mathrm{MHz}$

The quasi-static magnetic field and the thermal plasma properties were calculated in the framework of EUROfusion integrated modelling (EU-IM) using the METIS code which is described in [32]. To determine the input temperature and density profiles, METIS uses fitted JET experimental data from CRONOS fitting tools [33], using the high resolution thomson scattering (HRTS) for the electron and density temperature profiles, the charge exchange (CX) for the ion temperature profile and the visible spectroscopy (KS3) for the $Z_{\text {eff }}$ profile. Figure 3 shows a comparison between the fitted profiles with the experimental values. The plasma equilibrium is also given by METIS, illustrated in figure 2, ensuring that the resolution of the equilibrium and profiles are the same. 

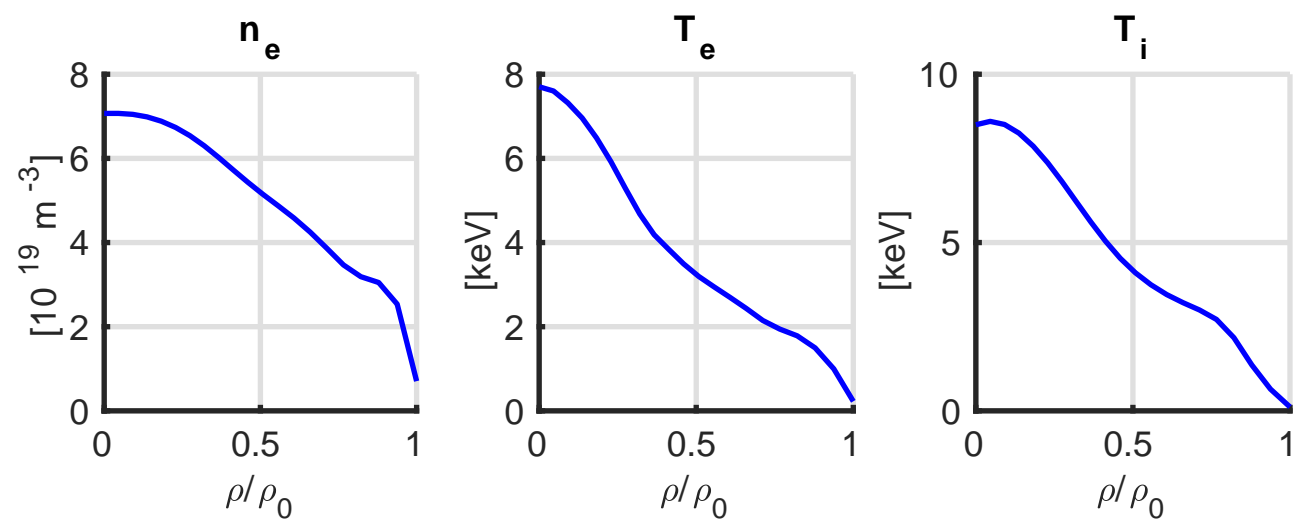

Figure 3: Input proiles of electron density (left) and electron (middle) and ion (right) temperature as a function of the normalized toroidal flux coordinate $\left(\rho=\sqrt{\Phi / \pi B_{0}}\right.$ where $\Phi$ is the toroidal magnetic flux, $B_{0}$ the vacuum magnetic field and $\rho_{0}$ the value of $\rho$ at the last closed flux surface) obtained by CRONOS fitting tool (blue line) for JET shot \#86614

\section{Modelling of NBI and ICRH heating of JET shot \#86614}

\subsection{NBI source simulation}

First of all, we simulated the fast ion source created by NBI in the collisional stationary state (i.e $\Delta t=0.3 s)$ with NEMO / SPOT and the results are presented in figure 4. The results show that $72 \%$ of NBI power is transferred to the ions and $27 \%$ to the electron with $1 \%$ of fast ion losses. The NBI power deposition is maximum at $\rho / \rho_{0} \simeq 0.2$. This heating produces a fast $\mathrm{D}$ fraction of $5 \%$ (in density) having a central temperature of $40 \mathrm{keV}$ about 4 times greater than the thermal temperature. The fast D temperature profile has its maximum at the plasma centre. This location of the NBI deposition is an advantage for the NBI and ICRH synergy because the ICRH resonance is located near the plasma centre.

In addition, NBI heating allows increasing the D-D reaction rate by $14 \%$ due to the beam-target reaction. The simulation gives a neutron rate of $R_{D D}=1.8 \cdot 10^{16} \mathrm{~m}^{-3} \cdot \mathrm{s}^{-1}$ corresponding to $20 \%$ less than the neutron rate found experimentally.
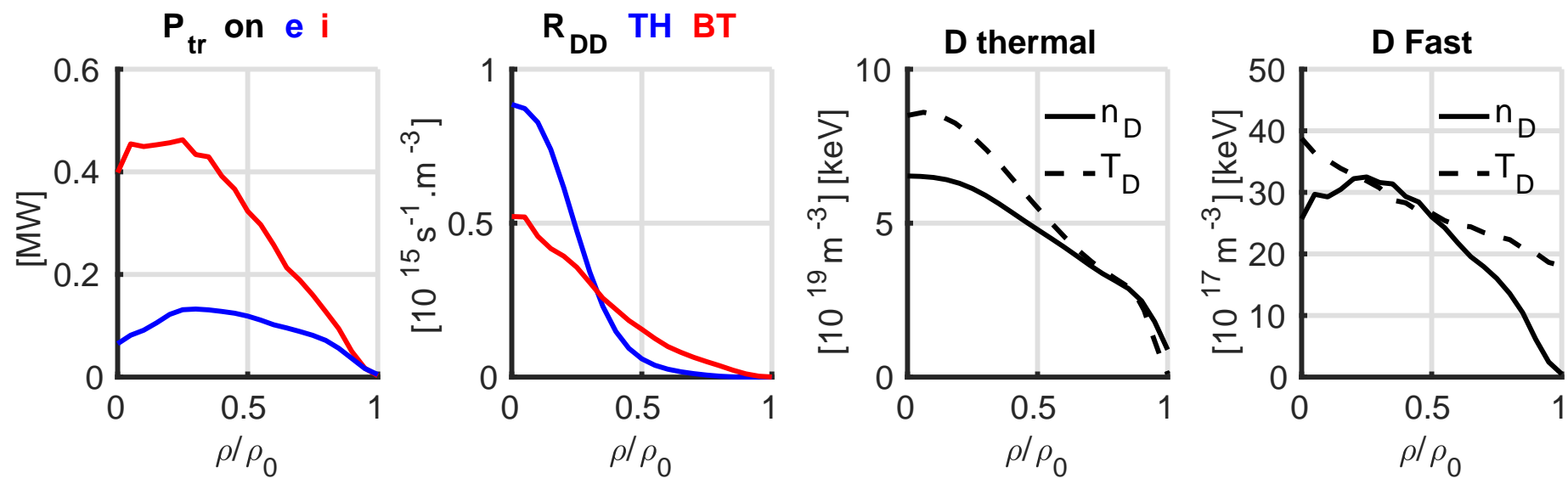

Figure 4: Results of NBI source simulation with NEMO/SPOT. In order from left to right : profile of transfered power on electrons (in blue) and on ions (in red), profile of neutron rate for thermal reactions (in blue) and beam-target reactions (in red), profile of density (in lines) and temperature (in dash) of thermal deuterium, profile of density (in lines) and temperature (in dash) of fast deuterium $\left(T_{\text {D fast }}=2 / 3 T_{\perp}+1 / 3 T_{\|}\right)$

As explained in section 2, we use a bi-maxwelian fit (cf eq.3) to represent the fast D distribution (cf eq.4). Figure 5 presents a comparison between the fit and the distribution given by SPOT, showing the typical accuracy obtained with the bi-Maxwellian fit. The graph of figure 5 on the right shows two curves because the NBI heating produces a co-current which makes the distribution in the parallel direction asymmetrical. 

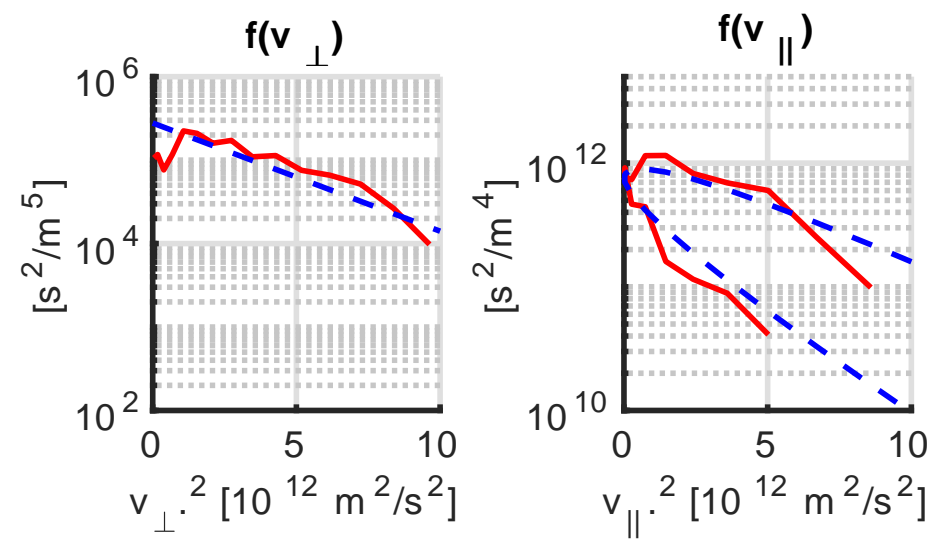

Figure 5: Comparaison between the SPOT distribution (in solid red) where $f\left(v_{\perp}\right)=\sum_{k} p_{k} / 2 \pi v_{\perp k} k \in\left[v_{\perp i} ; v_{\perp i+1}\right]$ and $f\left(v_{\|}\right)=\sum_{m} p_{m} m \in\left[v_{\| i} ; v_{\| i+1}\right]$ with $\mathrm{p}$ being the weight of the particle and the bi-maxwellian fit (in dash blue) in perpendicular and parallel direction at $\rho / \rho_{0}=0.1$, where $f\left(v_{\perp}\right)=n_{f}\left(\frac{m_{f}}{2 \pi T_{\perp}}\right) \exp \left(-\frac{m_{f} v_{\perp}^{2}}{2 T_{\perp}}\right)$ and $f\left(v_{\|}\right)=$ $n_{f}\left(\frac{m_{f}}{2 \pi T_{\|}}\right)^{1 / 2} \exp \left(-\frac{m_{f} v_{\|}^{2}}{2 T_{\|}}\right)$with $T_{\perp}$ and $T_{\|}$calculated by equations (4).

Since we use a Monte Carlo code to solve the Fokker-Planck equation, we must make sure that the number of particles used in SPOT to represent the distribution function is sufficient to get a reasonable calculation time and to avoid statistical fluctuations on the profiles. For this purpose, we compare three simulations with respectively 1000, 10000 and 100000 particles. Figure 6 presents the profiles of the temperature and the density of D beams, as well as its distribution for these simulations. The results shows to get a numerically stable solution for the D distribution function. Using 10000 particles appears to be a good compromise, since going to 100000 does not improve much the accuracy of the solution, while with 1000 particles only a different and fluctuating solution is obtained (cf table 2). In the rest of our study, we will therefore use 10000 particles for the Monte Carlo code statistics.
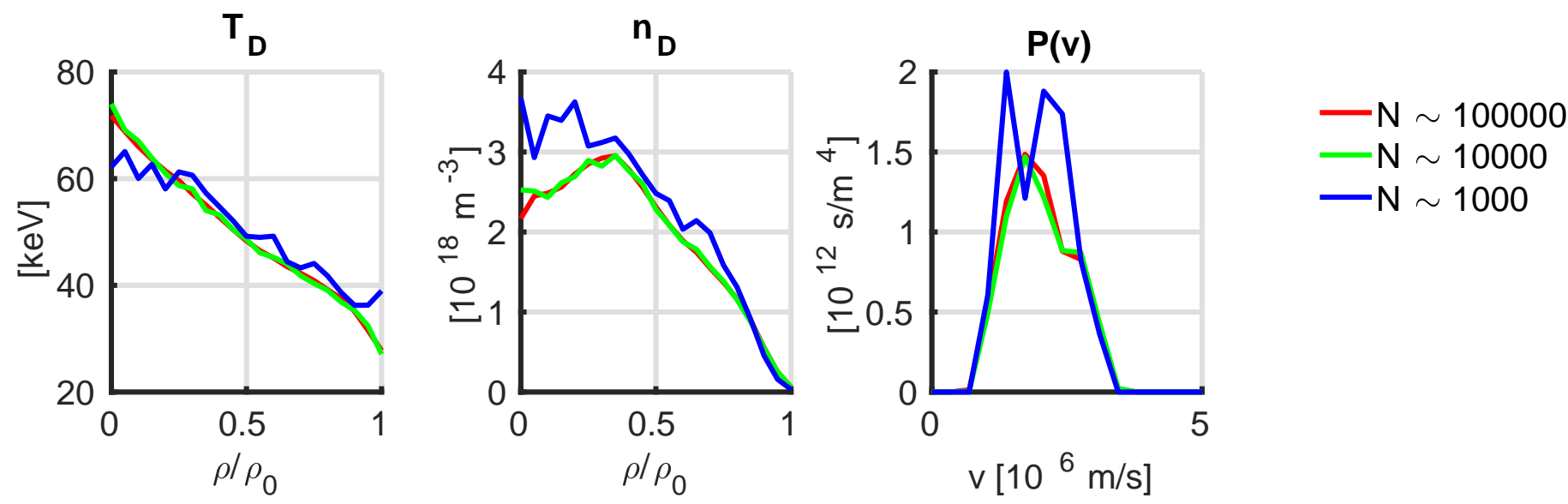

Figure 6: Comparison of temperature and density of $\mathrm{D}$ beam profiles and $\mathrm{D}$ beam distribution in terms of Monte-Carlo code statistic $\mathrm{N}$, for $N \sim 100000$ (in red), $N \sim 10000$ (in green), $N \sim 1000$ (in blue)

\begin{tabular}{|c|c|c|c|}
\hline $\mathrm{N}$ & 100000 & 10000 & 1000 \\
\hline$W_{D} M J$ & 0.74 & 0.74 & 0.87 \\
\hline$n_{D} 10^{18} m^{-3}$ & 1.56 & 1.56 & 1.75 \\
\hline
\end{tabular}

Table 2: Summary of total energy content and density of $\mathrm{D}$ beam for the three simulations 


\subsection{Self-consistent simulation of NBI+ICRH synergy}

In a second step, we simulated the propagation and the absorption of the ICRF wave with the EVE/SPOT code package. As described in section 2, several iterations between the full wave code and the Fokker-Planck code are necessary to reach the stationary state of the wave and the fast $\mathrm{D}$ distribution.

Figure 7 shows the evolution of the global quantities of the plasma of self-consistent simulation EVE/SPOT for a plasma with $2 \%$ of $\mathrm{H}$ concentration. In this figure SPOT carries out $50 \mathrm{~ms}$ of calculation between each iteration with EVE, so iterations between the two codes occur approximately 10 times faster than the relaxation time of the distribution function. Indeed the figure shows that after $500 \mathrm{~ms}$ (10 iterations), the distribution function and all parameters have reached a stationary state. Up to 40 iterations ( $2.0 \mathrm{~s}$ of simulation) have been carried out to make sure that the quantities do not evolve anymore (apart from small statistical fluctuations) and we averaged the global quantities on the tenth and last iteration represented by the black dashes of the figure 7 .

In the fully converged solution, the first plot shows that $0.90 \mathrm{MW}$ of ICRH power is absorbed by fast D and the rest is absorbed by thermal $\mathrm{H}$ and $\mathrm{D}$. The second plot shows that $66 \%$ of total power heating is transferred to the ions, $33 \%$ to the electrons and $1 \%$ of fast ions losses. The evolution observed results in an increase of the ICRH absorption by the fast $\mathrm{D}$. The third plot shows that the neutron rate of D-D reaction reaches $2.1 \cdot 10^{16} \mathrm{~s}^{-1}$ with NBI and ICRH synergy which is very close to the experimental value that is of $2 \cdot 10^{16} \mathrm{~s}^{-1}$. Uncertainties on $T_{i}$, arising from measurements or equilibrium reconstruction [30], have been taken into account by performing an alternative calculation increasing $T_{i}$ by $12 \%$. Proceeding like this, an increase of $17 \%$ is obtained in the neutron rate. The changes are not due to differences in the synergy (the distribution functions are unaffected), but to changes in the thermal neutron rate (35\%) and to changes in the reaction rates of fast $\mathrm{D}$ ions with thermal ions (1.5\%), as shown in the figure 8 comparing the profiles obtained between the simulation with the Ti input profile described above (in pointless) and the simulation with the Ti input profile increased by $12 \%$ (in dash). The last plot shows that the energetic content of fast deuterium in perpendicular direction $\left(W_{D \perp}=0.77 M J\right)$ is larger than in parallel direction $\left(W_{D \|}=0.27 M J\right)$.

We now compare the fully converged solution after $500 \mathrm{~ms}$ with the situation after only $50 \mathrm{~ms}$ of SPOT calculation, i.e. the first time slice of the simulation where ICRH effects are accounted for in the SPOT output. We find $39 \%$ of difference on the absorbed ICRH power, $4 \%$ of difference on power transferred to the bulk electrons and ions, $12 \%$ of difference on neutron rate, $3 \%$ on fast $\mathrm{D}$ density and $6 \%$ of difference for the parallel and perpendicular energetic content of $\mathrm{D}$ fast. These differences quantify the importance of simulating the self-consistent fast D distribution until it has reached its stationary state.
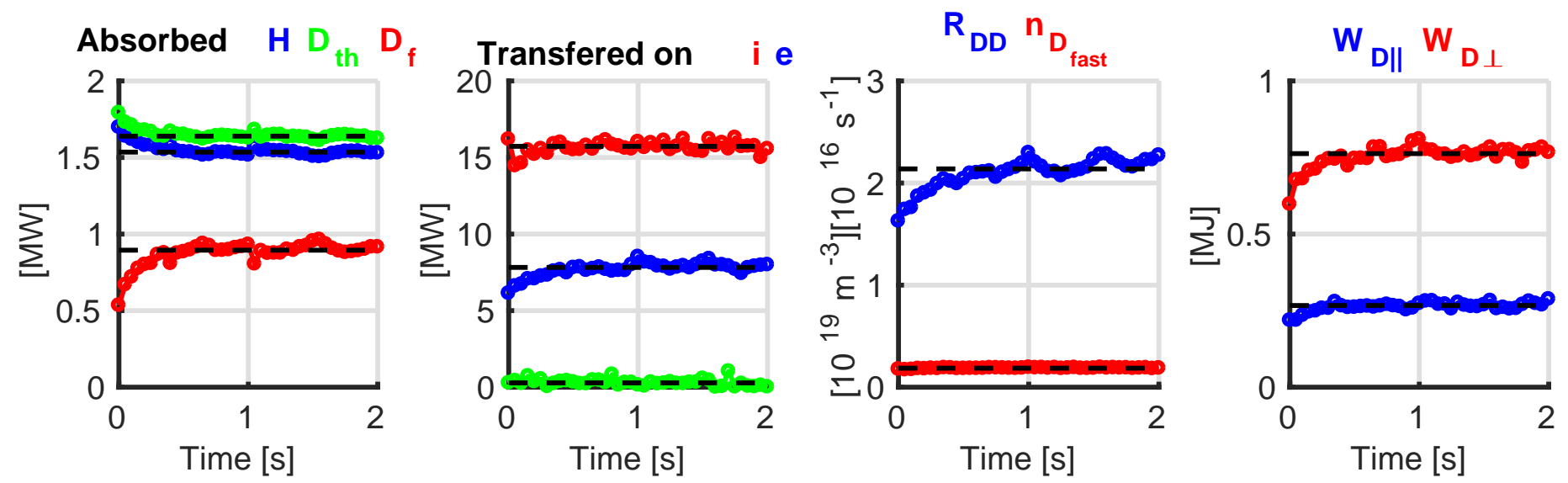

Figure 7: Evolution of the global quantities of the plasma as a function of the simulation time between EVE and SPOT with $2 \%$ of $\mathrm{H}$. In order from left to right : evolution of ICRH power absorbed by H (in blue), thermal D (in green) and fast D (in red), evolution of NBI and ICRH power transferred to ions (in red) and electrons (in blue) with fast ion loss (in green), evolution of neutron rate of D-D reaction (in blue) and fast D density (in red), evolution of energetic content of fast $\mathrm{D}$ in perpendicular (in red) and parallel (in blue) direction. 

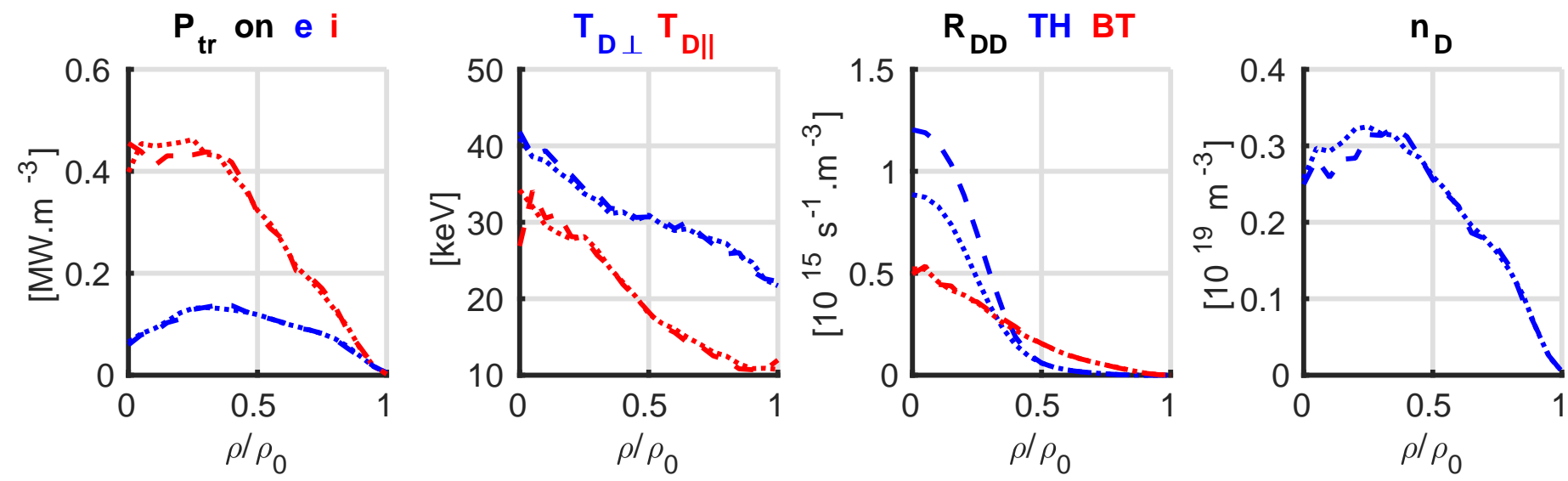

Figure 8: Comparison of the profiles obtained between the simulation with the Ti input profile described above (dotted) and the simulation with the Ti input profile increased by $12 \%$ (dashed). In order from left to right : profiles of NBI power transfered on electrons (in blue) and on ions (in red), profiles of effective temperatures parallele and perpendicular of fast $\mathrm{D}$, profile of neutron rate for thermal reactions (in blue) and beam-target reactions (in red) and profile of $\mathrm{D}$ fast density.

In order to characterize the influence of the self-consistent coupling on the $500 \mathrm{~ms}$ time scale, the self-consistent simulation is compared with a modelling of fast distribution evolution with only SPOT (i.e without iteration between EVE and SPOT). Figure 9, shows the comparison of the evolution of the global quantities of the plasma between the two simulations. Significant differences appear on the ICRH power absorbed by the fast D, as well as a difference on neutron rate of $\mathrm{D}-\mathrm{D}$ reaction and on the perpendicular energetic content of fast $\mathrm{D}$. The quantities and differences are sumarized in table 3 .
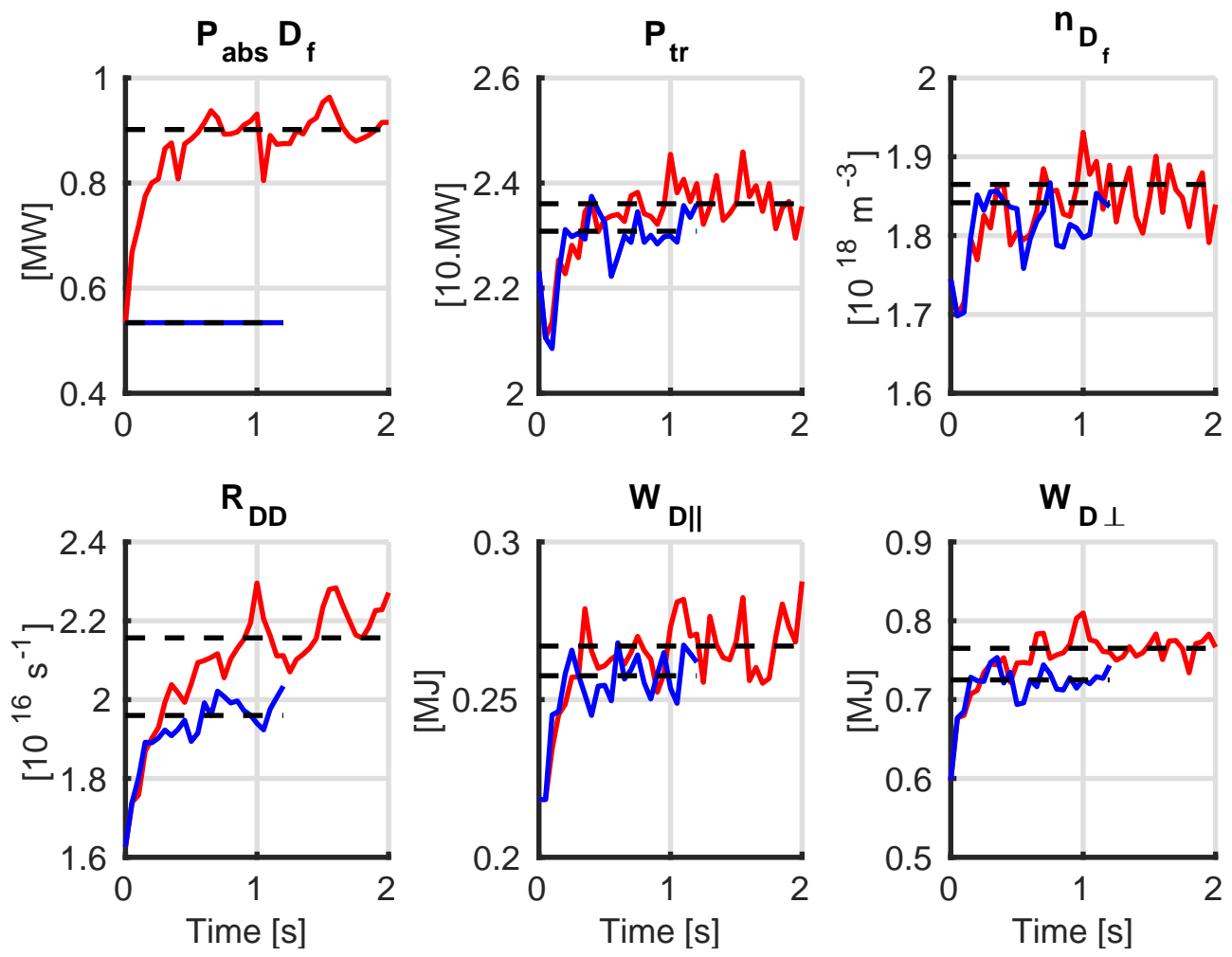

Figure 9: Comparison of evolution of the global quantities of the plasma. The red lines represent the simulation with self-consistent iterations every $50 \mathrm{~ms}$ between EVE and SPOT. The blue lines represent the SPOT simulation without iteration with EVE. Top in order from left to right : evolution of ICRH power absorbed by D fast, evolution of NBI + ICRH power transferred to bulk plasma, evolution of $\mathrm{D}$ fast density. Down in order from left to right : evolution of neutron rate of D-D reaction, evolution of parallel energy content of $\mathrm{D}$ fast and perpendicular. 


\begin{tabular}{|c|c|c|c|c|c|c|c|}
\hline Iteration & $P_{a b s} D_{f a} M W$ & $P_{a b s} D_{t h} M W$ & $P_{a b s} H M W$ & $n_{D f a} 10^{18} m^{-3}$ & $R_{D D} 10^{16} s^{-1}$ & $W_{D \perp} M J$ & $W_{D \|} M J$ \\
\hline \hline yes & 0.90 & 1.64 & 1.53 & 1.86 & 2.16 & 0.77 & 0.27 \\
\hline no & 0.53 & 1.79 & 1.70 & 1.84 & 1.96 & 0.73 & 0.26 \\
\hline differences & $40.8 \%$ & $9.4 \%$ & $10.9 \%$ & $1.1 \%$ & $9.2 \%$ & $5.2 \%$ & $3.7 \%$ \\
\hline
\end{tabular}

Table 3: Summary of the simulation with self-consistent iterations every $50 \mathrm{~ms}$ between EVE and SPOT and without iterations

Figure 10 presents the differences on profiles obtained between the simulation with self-consistent iterations every $50 \mathrm{~ms}$ between EVE and SPOT and the SPOT simulation without iterations with EVE. The changes are located in the region $\rho / \rho_{0}<0.3$, which is the region in which the ICRH D resonance and the $\mathrm{D}$ beam deposition overlap. The perpendicular effective temperature increase is more important in the self-consistent case whereas the parallel effective temperature does not change, which is consistent with the ICRH physical process, since the diffusion coefficient only includes the perpendicular component of the ion velocity (cf eq.5). We have observed (fig.10) that the evolution of the fast D distribution occurs essentially close to the ICRH absorption, i.e. $\rho / \rho_{0}<0.3$.
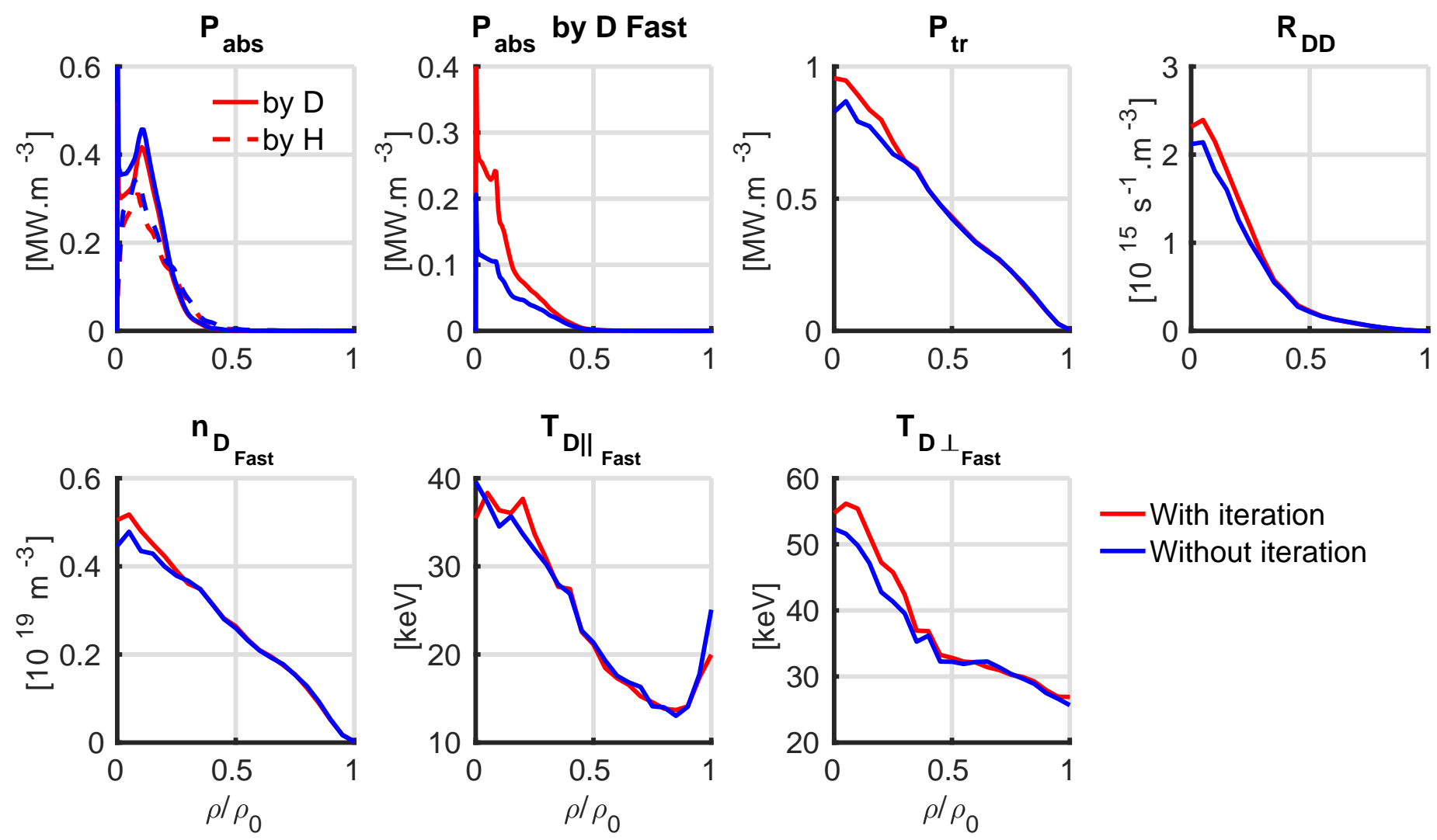

Figure 10: Comparison of the profiles obtained between the simulation with self-consistent iterations every 50 ms between EVE and SPOT (in red) and the SPOT simulation without iterations with EVE (in blue). Top in order from left to right : profiles of power absorbed by $\mathrm{H}$, thermal $\mathrm{D}$ and fast $\mathrm{D}$, profiles of power transfered on bulk plasma and profiles of neutron rate of D-D reaction. Down in order from left to right : profiles of fast D density, profiles of effective temperatures parallele and perpendicular of fast $\mathrm{D}$.

Figure 11 presents the fast $\mathrm{D}$ distribution at $\rho / \rho_{0}=0.1$ in the perpendicular and parallel direction obtained by the simulation with self-consistent iterations every $50 \mathrm{~ms}$ between EVE and SPOT and the SPOT simulation without iterations with EVE. We see that the high energy tail above $v_{\perp}>3.10 \mathrm{~m} . \mathrm{s}^{-1}$ develops only when modelling self-consistently the wave and the fast $\mathrm{D}$ distribution. Figure 12 presents the real part of positive and negative component of the electric field of the ICRF wave for the simulation with self-consistent iterations every $50 \mathrm{~ms}$ between EVE and SPOT and the SPOT simulation without iterations with EVE in the equatorial plane. This result shows that iterations modify the electric field amplitude by $10 \%$ at the resonance of the ICRF wave in the equatorial plane, as in the poloidal plane as shown by the figure 13. Nevertheless, besides this small evolution of the electric field amplitude, the structure of the electric field 
pattern in the poloidal plane remains globally unchanged by the evolution of the fast D distribution.
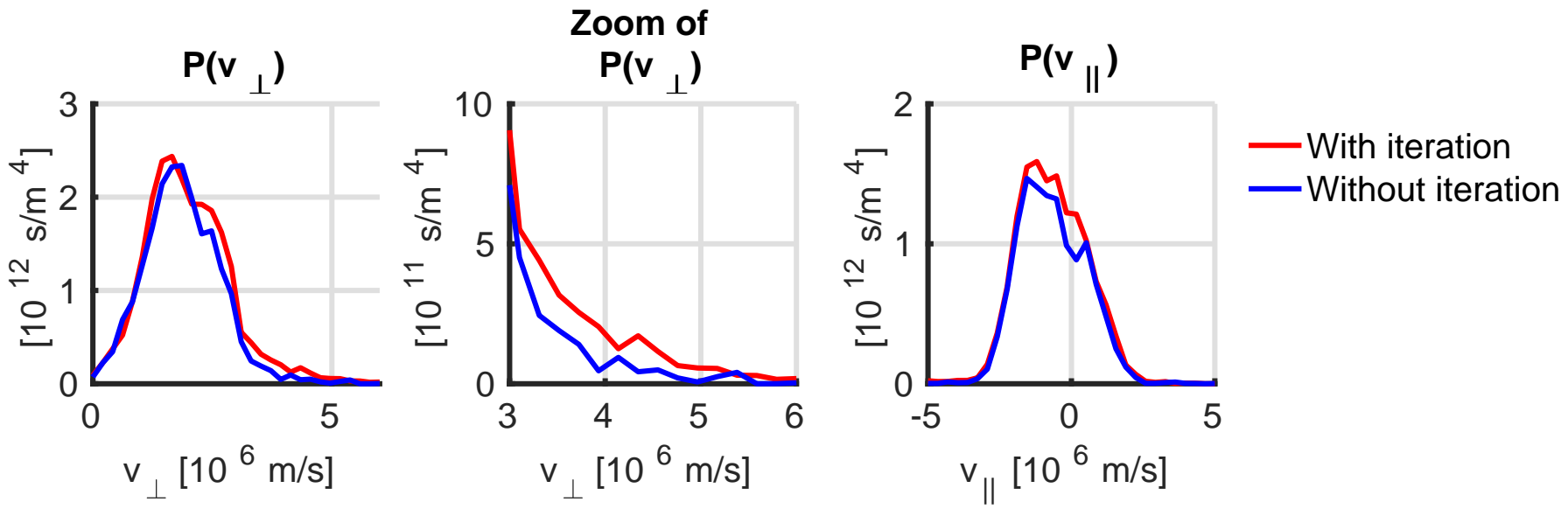

Figure 11: Comparison of the distributions perpendicular (left) and parallel (right) of the fast $\mathrm{D}$ to $\rho / \rho_{0}=0.1$ between the simulation with self-consistent iterations every $50 \mathrm{~ms}$ between EVE and SPOT (in red) and the SPOT simulation without iterations with EVE (in blue). Where $P\left(v_{\perp}\right)=\sum_{k} p_{k} k \in\left[v_{\perp i} ; v_{\perp i+1}\right]$ and $P\left(v_{\|}\right)=\sum_{m} p_{m} m \in\left[v_{\| i} ; v_{\| i+1}\right]$ with $\mathrm{p}$ being the weight of the particle.
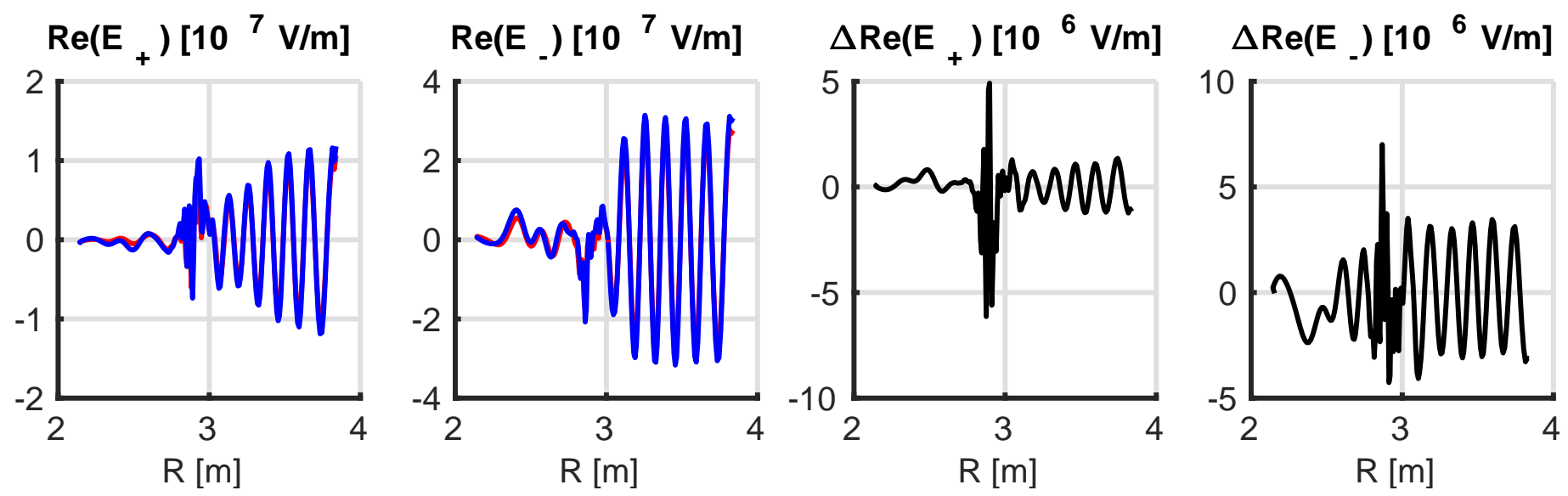

Figure 12: Comparison of real part of the positive component of the electric field of the ICRF wave (first) and negative (second) between the simulation with self-consistent iterations every $50 \mathrm{~ms}$ between EVE and SPOT (in red) and the SPOT simulation without iterations with EVE (in blue) in the equatorial plane. The last ones plot represent the difference between the both simulations 


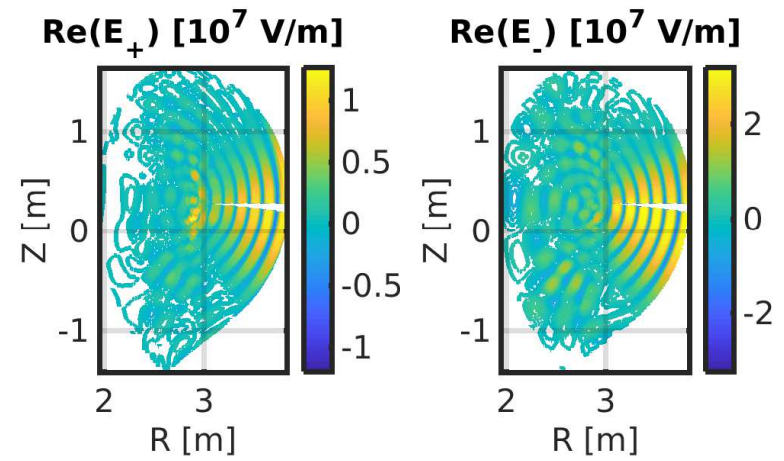

(a) EVE modelling
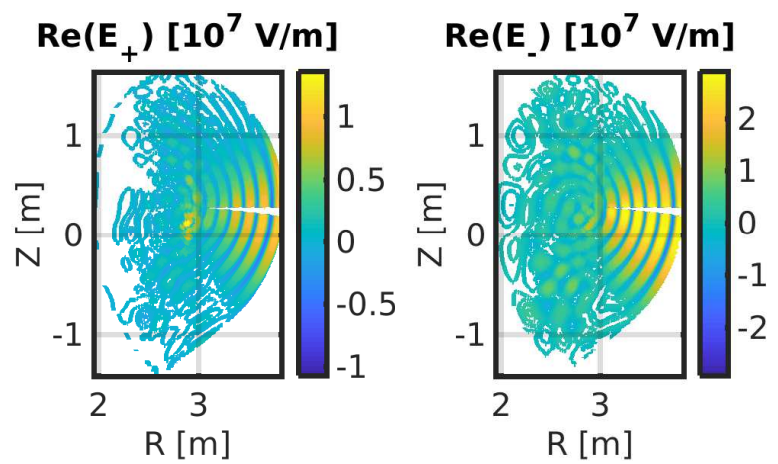

(b) EVE/SPOT modelling
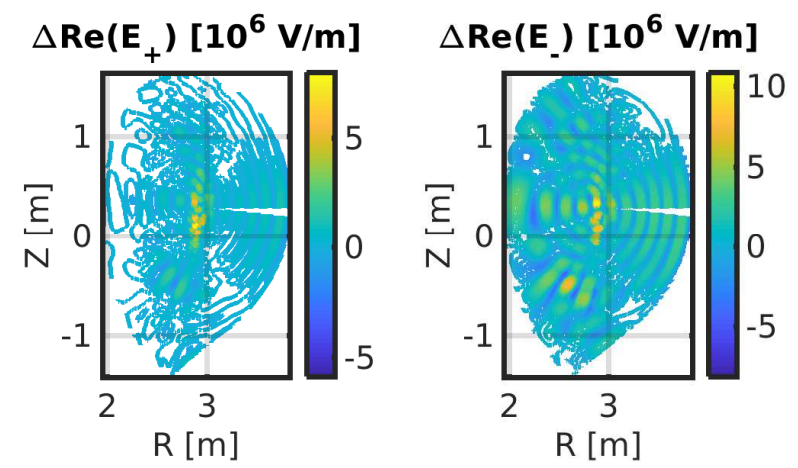

(c) Difference

Figure 13: Comparison of real part of the positive component of the electric field of the ICRF wave (left) and negative (right) between the simulation with self-consistent iterations every $50 \mathrm{~ms}$ between EVE and SPOT (13a) and the SPOT simulation without iterations with EVE (13b) and the difference (13c).

The comparison between the simulations with and without iterations between EVE and SPOT shows that it is necessary to take into account the feedback of the fast $\mathrm{D}$ distribution on the propagation and absorption of the ICRF wave. Indeed, if this feedback is not modeled, the simulation does not model accurately the high energy tail in the distribution of fast $\mathrm{D}$, which impacts the determination of the neutron rate as well as the propagation and the absorption of the ICRF wave. The self-consistent simulation enables to capture the evolution of the D distribution function, resulting in an increase of the fast $\mathrm{D}$ density, an increase of the ICRH power absorbed by this population and an higher fast $\mathrm{D}$ energy tail. The self-consistent NBI+ICRH simulation enables to recover the experimental value of the neutron rate within $5 \%$.

\subsection{Comparison between NBI heating only and NBI+ICRH synergy}

In order to characterize the impact of the heating scheme on the fast D distribution, we compare the NBI-only heating and the combined NBI and ICRH with equivalent power. For this purpose, we compare the results presented in the previous section of the NBI and ICRH synergy with 22MW of NBI and 5MW of ICRH with two simulations with pure NBI heating, made with NEMO/SPOT with respectively 27 and $22 \mathrm{MW}$ of NBI power.

Figure 14 presents the profiles, obtained by these three heating schemes, of the power transferred to the plasma of the effective temperatures of the fast $\mathrm{D}$, the neutron rate of the D-D reaction and the fast $\mathrm{D}$ density. With the NBI and ICRH synergy the power transferred to the electrons is larger than in the case of pure NBI heating, as well as the power density transferred to the ions close to the plasma center. This difference is due to the fact that NBI and ICRH synergy produces ions that are more energetic than pure NBI heating. In case of pure NBI heating the parallel and perpendicular temperatures of the fast D remain the same while increasing the NBI power, whereas with the NBI and ICRH synergy, the perpendicular effective temperature is higher and increases by $28 \%$ in the center. However, increasing NBI power can increase the fast D density by $17 \%$ while adding ICRH heating to NBI heating can increase fast density by only $5 \%$. But by increasing the perpendicular temperature, the synergy makes it possible to increase by $15 \%$ the neutron rate of the D-D reaction with respect to the NBI heating at equivalent power. Pure NBI heating increases the fast D density over the entire plasma volume (due to source increase) whereas the NBI+ICRH scheme increases the fast density only inside $\rho / \rho_{0}=0.4$ (kinetic effect of the synergy). 
Figure 15 presents the perpendicular and parallel distribution at $\rho / \rho_{0}=0.1$ of fast D obtained between the NBI modelling with a power of $27 \mathrm{MW}$, NBI modelling with a power of $22 \mathrm{MW}$ and NBI (22MW) + ICRH (5MW) modelling. This comparison shows that the NBI + ICRH synergy modifies the distribution of the heated ion by increasing the tail at high energy, whereas the increase in the power of the NBI heating induces no change in shape of the distribution.
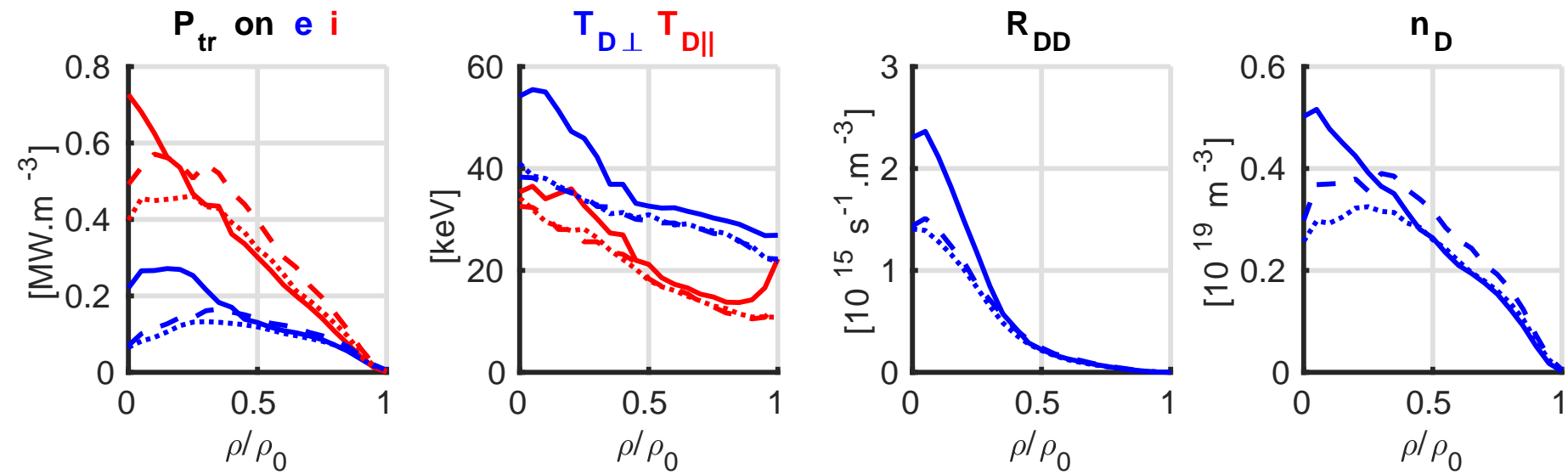

Figure 14: Comparison of the profiles obtained between the NBI model with a power of 27MW (dash), the modelling NBI $(22 \mathrm{MW})+\mathrm{ICRH}(5 \mathrm{MW})$ (solid line) and the modelization NBI with a power of 22MW (point). In order from left to : profiles of the power transferred to ions (in red) and electrons (in blue), profiles of the effective perpendicular (blue) and parallel (red) temperature of the fast $\mathrm{D}$, profile of the neutron rate of the $\mathrm{D}-\mathrm{D}$ reaction, profile of the fast $\mathrm{D}$ density.
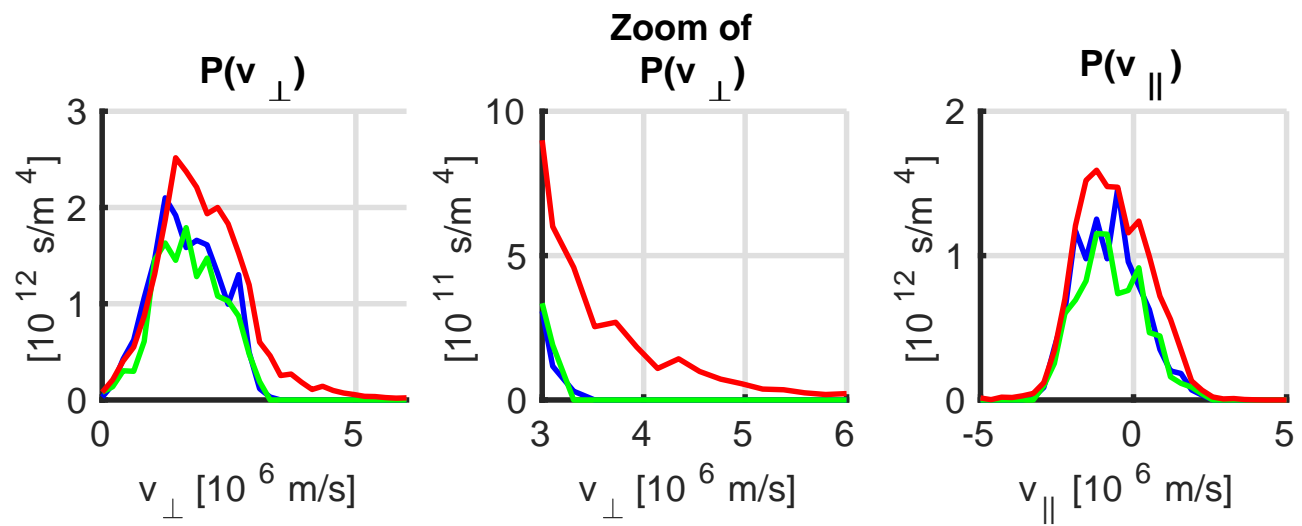

Figure 15: Comparison of distributions in the perpendicular (left) and parallel (right) direction of the fast D at $\rho / \rho_{0}=0.1$ obtained between the NBI modelling with a power of $27 \mathrm{MW}$ (in blue), NBI modelling with a power of $22 \mathrm{MW}$ (in green) and NBI $(22 \mathrm{MW})+$ ICRH $(5 \mathrm{MW})$ (in red) modelling.

The results therefore show that in order to increase the production of fusion reactions, it is more efficient to use combined NBI and ICRH than NBI only since the synergy yields fast ions with a higher energy for equivalent heating for a given total auxiliary heating power. Moreover thanks to the ICRH heating, the increase of the fast ion density as well as its energetic content is more localized in the core of the plasma $\left(\rho / \rho_{0}<0.4\right)$.

\section{Impact of minority species concentration}

In this section we study the impact of minority species concentration on NBI and ICRH synergy. The dominant impact of the increase from 1 to $6 \%$ of the concentration of the minority species is the increase of the ICRH power absorption on the Hydrogen (minority species), resulting in a decrease of the remaining power to be transferred to the fast D (cf tab.4). This modification impacts the NBI and ICRH synergy by decreasing the density and the energetic content of the fast D and the rate of DD reaction. 


\begin{tabular}{|c|c|c|c|c|c|c|c|}
\hline$[\mathrm{H}] \%$ & $P_{a b s} D_{f a} M W$ & $P_{a b s} D_{t h} M W$ & $P_{a b s} H M W$ & $n_{D f a} 10^{18} m^{-3}$ & $R_{D D} 10^{16} s^{-1}$ & $W_{D \perp} M J$ & $W_{D \|} M J$ \\
\hline \hline 1 & 1.16 & 2.00 & 0.93 & 1.92 & 2.39 & 0.80 & 0.28 \\
\hline 2 & 0.90 & 1.64 & 1.53 & 1.86 & 2.16 & 0.77 & 0.27 \\
\hline 4 & 0.62 & 1.17 & 2.26 & 1.84 & 1.95 & 0.73 & 0.26 \\
\hline 6 & 0.53 & 0.92 & 2.58 & 1.80 & 1.86 & 0.71 & 0.25 \\
\hline
\end{tabular}

Table 4: Summary of self-consitent result for different concentration of H. $P_{a b s} D_{f a}, P_{a b s} D_{t h}$ and $P_{a b s} H$ are respectively the ICRH power absorbed by the fast D, the thermal D and H. $n_{D f a}$ is the density of fast D. $R_{D D}$ is the neutron rate of DD reaction. $W_{D \perp}$ and $W_{D \|}$ are respectively the energetic content in perpendicular and parallel direction of fast $\mathrm{D}$.

Figure 16 presents a comparison of the evolution of the global quantities of the plasma according to the time simulation with self-consistent iterations every $50 \mathrm{~ms}$ between EVE and SPOT for different concentration of $\mathrm{H}(1 \%, 2 \%, 4 \%, 6 \%)$. This result shows that the time necessary to reach the stationary state of NBI and ICRH synergy does not depend on the concentration of the minority species and all simulations reach the stationary state after 0.5 seconds, which corresponds to 10 iterations between EVE/SPOT.

The impact of the concentration of minority species on NBI and ICRH synergy is not proportional between the ICRH power absorbed by the $\mathrm{D}$ fast, the characteristics of $\mathrm{D}$ fast and the neutron rate of $\mathrm{DD}$ reaction, due to the non-linear nature of on NBI and ICRH synergy. Between simulations at $1 \%$ and $4 \%$ of $\mathrm{H}$, there is an increase of $50 \%$ for the ICRH power absorbed by the fast $\mathrm{D}$, of a few $\%$ for the power transferred to the bulk, the density and the parallel energy content of D fast, of $10 \%$ for the perpendicular energy content of the fast $\mathrm{D}$ and of $25 \%$ for the rates of neutron of the reaction DD.

On the other hand, between 4 and $6 \%$ of $\mathrm{H}$, a certain stability in the ICRH power distribution between the different ions of plasma is achieved, which explains the lower sensitivity of the different quantities.

These results show a slight decrease of the power transferred to the plasma bulk and of the fast $\mathrm{D}$ density because the simulations take into account the NBI power at the beginning. The changes resulting of the variation of the $\mathrm{H}$ minority concentration are localised mainly inside $\rho / \rho_{0}<0.2$, as shown in figure 17 which presents a comparison of the profiles obtained for different concentration of $\mathrm{H}(1 \%, 2 \%, 4 \%, 6 \%)$.

Figure 18 presents a comparison of the distributions perpendicular and parallel of the fast $\mathrm{D}$ to $\rho / \rho_{0}=0.1$ obtained for different concentration of $\mathrm{H}(1 \%, 2 \%, 4 \%, 6 \%)$. These results show that increasing the concentration of $\mathrm{H}$ increases the high energy tail of the perpendicular distribution of the fast $\mathrm{D}$ but does not affect the parallel distribution. 

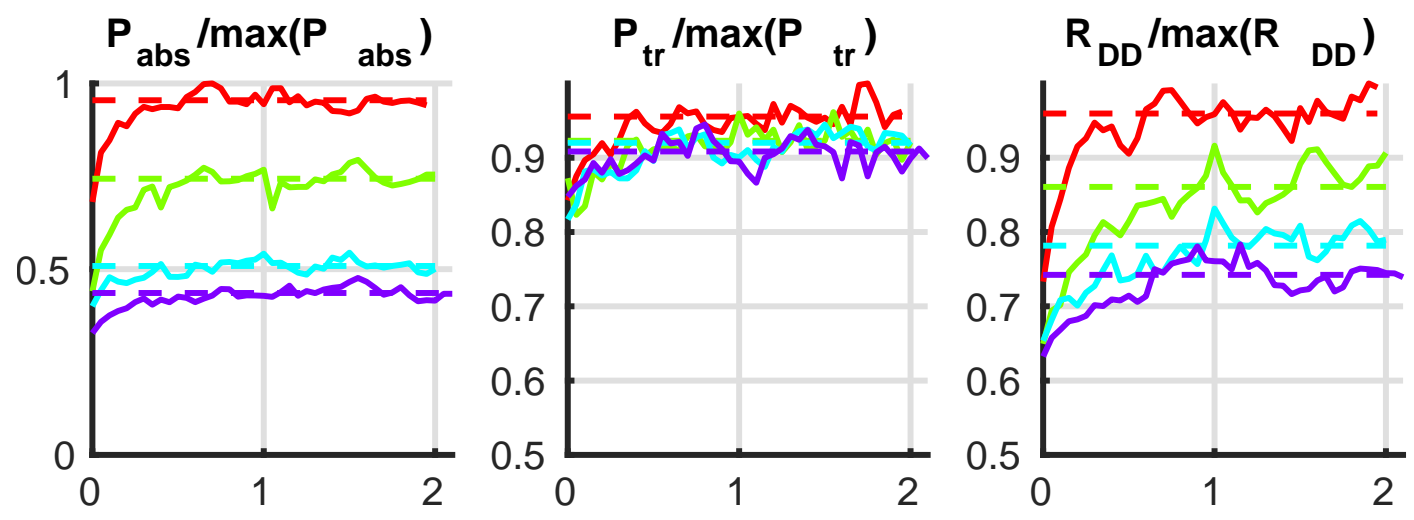

- $[\mathrm{H}]=1.0 \%$ $-[\mathrm{H}]=2.0 \%$ $[\mathrm{H}]=4.0 \%$

$[\mathrm{H}]=6.0 \%$
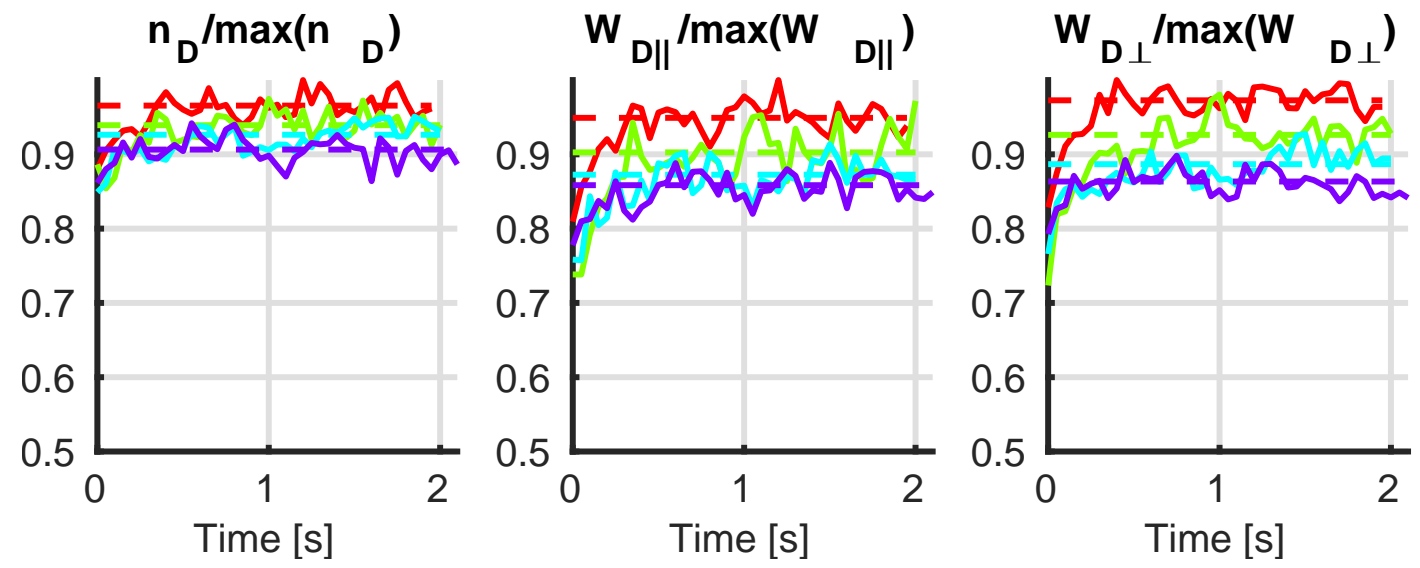

FigURE 16: Evolution of the global quantities of the plasma versus simulation time with self-consistent iterations every 50 ms between EVE and SPOT for different concentration of $\mathrm{H}$ (1\%, 2\%, 4\%, 6\%). Top in order from left to right : evolution of ICRH power absorbed by $\mathrm{D}$ fast, evolution of $\mathrm{NBI}+\mathrm{ICRH}$ power transferred to bulk plasma, evolution of neutron rate of D-D reaction. Down in order from left to right : evolution of D fast density, evolution of parallel energy content of D fast and perpendicular. All results are divided by the maximum of each quantities for the four simulations. 

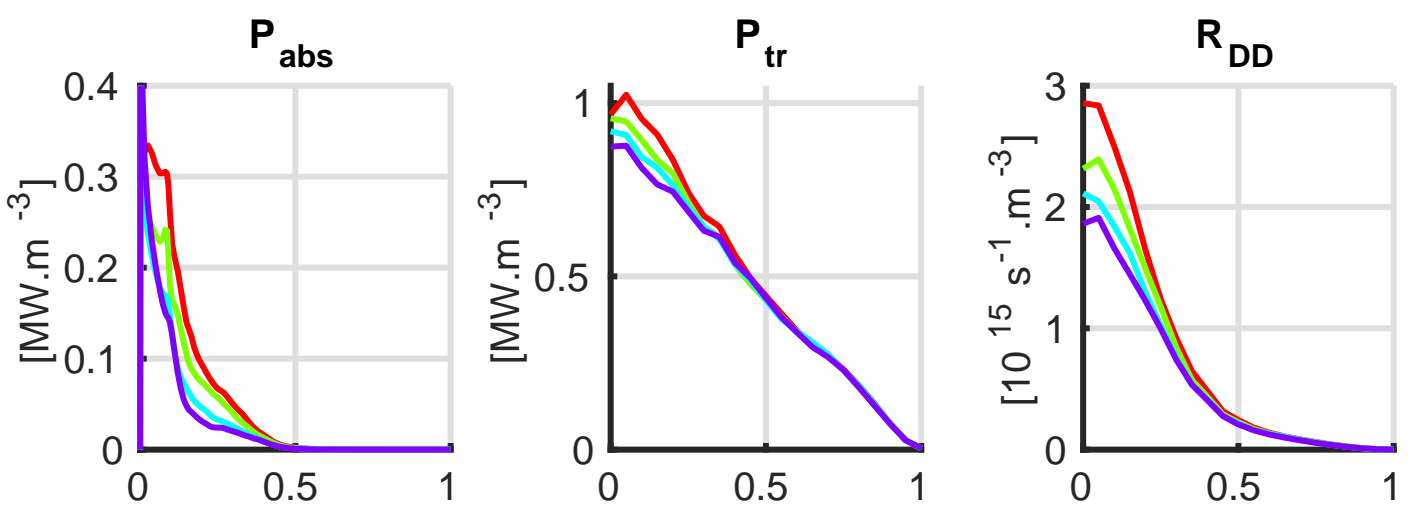

- $[\mathrm{H}]=1.0 \%$

$-[\mathrm{H}]=2.0 \%$

$\mathrm{H}]=4.0 \%$

$[\mathrm{H}]=6.0 \%$
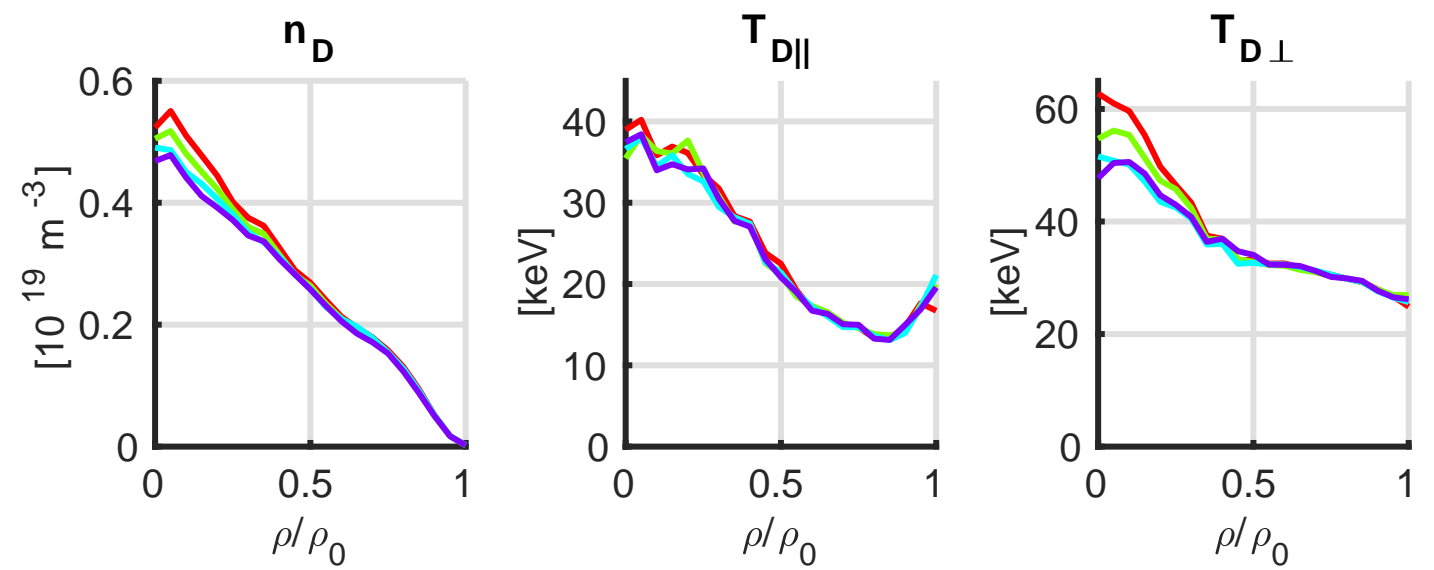

Figure 17: Comparison of the profiles obtained for different concentration of $\mathrm{H}(1 \%, 2 \%, 4 \%, 6 \%)$. Top in order from left to right : profiles of power absorbed by D fast, profile of power transfered on bulk plama by $\mathrm{D}$ and profiles of neutron rate of D-D reaction. Down in order from left to right : profiles of fast D density, profiles of effective temperatures parallel and perpendicular of fast $\mathrm{D}$.
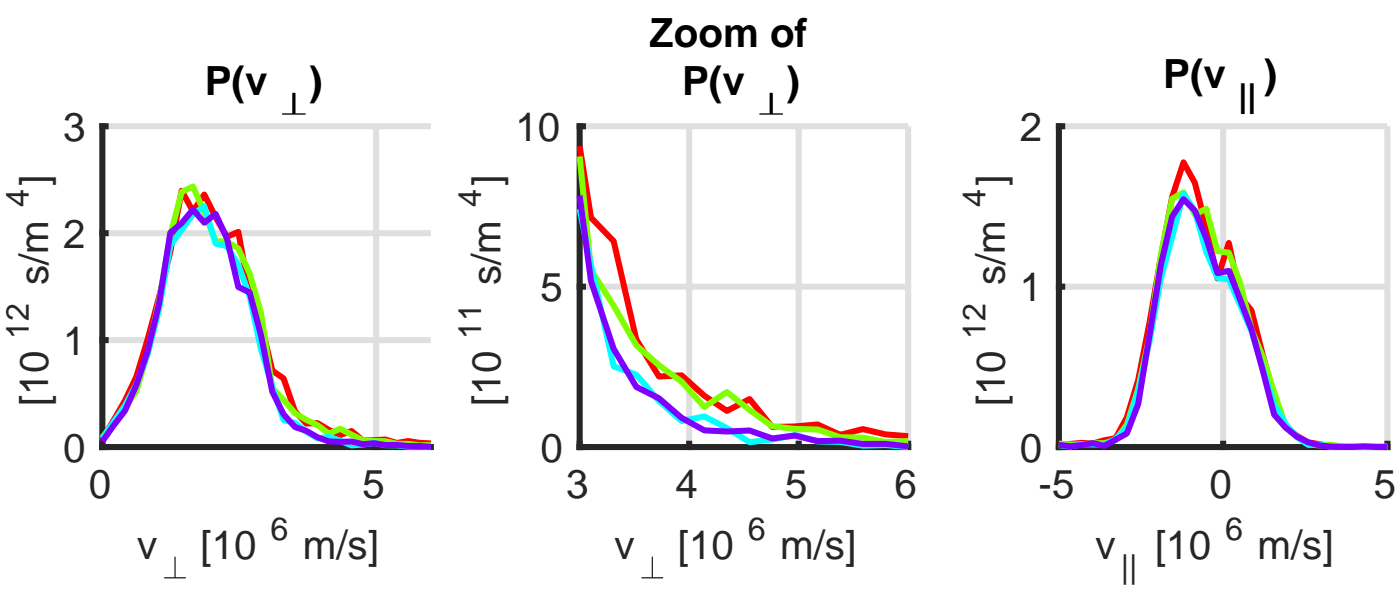

$[\mathrm{H}]=1.0 \%$

$-[\mathrm{H}]=2.0 \%$

$[\mathrm{H}]=4.0 \%$

$[\mathrm{H}]=6.0 \%$

Figure 18: Comparison of the distributions perpendicular (left) and parallel (right) of the fast D to $\rho / \rho_{0}=0.1$ obtained for different concentration of $\mathrm{H}(1 \%, 2 \%, 4 \%, 6 \%)$.

\section{Conclusions}

A new workflow for the self-consistent simulation of ICRH + NBI heating has been developed by coupling the full wave code EVE and the MonteCarlo orbit following code SPOT. One application of such development is the study of plasmas where 
a synergy between both heating schemes contributes to the creation of a significant fast ion tail which can potentially interact with other physical mechanisms such as turbulence or Alfvén modes. Towards the goal of self-consistent simulation of the full ion distribution function, a first step has been developed by using bi-Maxwellians distributions for representing the fast ion component heated by both heating schemes (here, the fast $\mathrm{D}$ ). This approach has been used to analyze a JET shot, \#86614, with significant ICRH, with a H minority scheme, and NBI D heating, for which the 2nd harmonic ICRH resonance coincides with the $\mathrm{H}$ one. The modelling performed shows that the interplay between both heating mechanisms is quite significant as the fast D perpendicular temperature obtained with only NBI, 40keV, increases up to $60 \mathrm{keV}$ due to this interplay. This has a strong impact on the neutron rate generation from D-D fusion reactions, which increases more than $20 \%$. The neutron rate calculated taking into account this self-consistent modelling of the ICRH-NBI synergy is in good agreement with the experimental measurement. The synergy effects are significant in this pulse because the NBI penetration for the JET hybrid scenario, to which the JET shot selects belongs, reaches far into the plasma core and the maximum of the fast ion source from NBI coincides with the ICRH deposition. However, such interplay strongly depends on the $\mathrm{H}$ concentration used, as above $2 \%$ the efficiency of such mechanism quickly decays due to the fact that $\mathrm{H}$ minority heating becomes the dominant scheme (with respect to the 2nd harmonic D heating).

Therefore, in order to maximize the DD reaction rate at JET, the control of the $\mathrm{H}$ concentration and the location of the ICRH resonance is essential for optimizing the synergetic effect on the fast D population pre-heated with NBI. The extrapolation of these results to DT plasmas, which is not straightforward because of the difference between the DD and DT cross-sections, will be performed in the future.

\section{Acknowledgement}

This work has been carried out within the framework of the EUROfusion Consortium and has received funding from the Euratom research and training programme 2014-2018 and 2019-2020 under grant agreement No 633053. The views and opinions expressed herein do not necessarily reflect those of the European Commission.

\section{References}

[1] M. J. Mantsinen, M.-L. Mayoral, V. G. Kiptily, S. E. Sharapov, B. Alper, A. Bickley, M. de Baar, L.-G. Eriksson, A. Gondhalekar, T. Hellsten, K. Lawson, F. Nguyen, J.-M. Noterdaeme, E. Righi, A. A. Tuccillo, and M. Zerbini. Alpha-Tail Production with Ion-Cyclotron-Resonance Heating of $\$ \wedge\{4\}$ He $\$-B e a m$ Ions in JET Plasmas. Phys. Rev. Lett., 88(10):105002, February 2002.

[2] H. Kimura, T. Fujii, M. Saigusa, S. Moriyama, K. Hamamatsu, M. Nemoto, and K. Tobita. High-harmonic ICRF heating experiments in JT-60. Plasma Phys. Control. Fusion, 35(7):845, 1993.

[3] W. W. Heidbrink, Y. Luo, K. H. Burrell, R. W. Harvey, R. I. Pinsker, and E. Ruskov. Measurements of fast-ion acceleration at cyclotron harmonics using Balmer-alpha spectroscopy. Plasma Phys. Control. Fusion, 49(9):1457, 2007.

[4] C. Hellesen, M. Gatu Johnson, E. Anderson Sundén, S. Conroy, G. Ericsson, E. Ronchi, H. Sjöstrand, M. Weiszflog, G. Gorini, M. Tardocchi, T. Johnson, V. G. Kiptily, S. D. Pinches, S. E. Sharapov, and JET-EFDA Contributors. Neutron emission generated by fast deuterons accelerated with ion cyclotron heating at JET. Nucl. Fusion, $50(2): 022001,2010$.

[5] D. Liu, W. W. Heidbrink, M. Podestà, R. E. Bell, E. D. Fredrickson, S. S. Medley, R. W. Harvey, and E. Ruskov. Profiles of fast ions that are accelerated by high harmonic fast waves in the National Spherical Torus Experiment. Plasma Phys. Control. Fusion, 52(2):025006, 2010.

[6] X. et al Litaudon. Overview of the JET results in support to ITER. Nucl. Fusion, 57(10):102001, 2017.

[7] Dani Gallart, Mervi Mantsinen, Clive Challis, Domenico Frigione, Jonathan Graves, Joerg Hobirk, Eva Belonohy, Agata Czarnecka, Jacob Eriksson, Marc Goniche, Carl Hellesen, Philippe Jacquet, Emmanuel Joffrin, Natalia Krawczyk, Damian King, Morten Lennholm, Ernesto Lerche, Ewa Pawelec, George Sips, Emilia Solano, Maximos Tsalas, and Marco Valisa. Modelling of combined ICRF and NBI heating in JET hybrid plasmas. EPJ Web Conf., 157:03015, 2017.

[8] R. Koch, G. Van Wassenove, D. Van Eester, H. Euringer, K. H. Finken, D. L. Hillis, and A. M. Messiaen. Interaction of ICRF waves with fast particles on TEXTOR. Plasma Phys. Control. Fusion, 37(11A):A291, 1995. 
[9] R. Bilato, M. Brambilla, O. Maj, L. D. Horton, C. F. Maggi, and J. Stober. Simulations of combined neutral beam injection and ion cyclotron heating with the TORIC-SSFPQL package. Nucl. Fusion, 51(10):103034, 2011.

[10] Žiga Štancar, Marina Gorelenkova, Sean Conroy, Jacob Eriksson, James Buchanan, and Luka Snoj. Generation of a plasma neutron source for Monte Carlo neutron transport calculations in the tokamak JET. Fusion Engineering and Design, April 2018.

[11] J. Garcia, C. Challis, J. Citrin, H. Doerk, G. Giruzzi, T. Görler, F. Jenko, P. Maget, and J. E. T. Contributors. Key impact of finite-beta and fast ions in core and edge tokamak regions for the transition to advanced scenarios. Nucl. Fusion, 55(5):053007, 2015.

[12] J. Garcia, T. Görler, and F. Jenko. Isotope and fast ions turbulence suppression effects: Consequences for high$\$\{\backslash$ Beta $\} \$$ ITER plasmas. Physics of Plasmas, 25(5):055902, March 2018.

[13] A. Di Siena, T. Görler, H. Doerk, E. Poli, and R. Bilato. Fast-ion stabilization of tokamak plasma turbulence. Nucl. Fusion, 58(5):054002, 2018.

[14] L.-G. Eriksson, T. Hellsten, and U. Willen. Comparison of time dependent simulations with experiments in ion cyclotron heated plasmas. Nucl. Fusion, 33(7):1037, 1993.

[15] L.-G. Eriksson and T. Hellsten. A model for calculating ICRH power deposition and velocity distribution. Phys. Scr., $52(1): 70,1995$.

[16] D. Gallart, M. J. Mantsinen, C. Challis, D. Frigione, J. Graves, E. Belonohy, F. Casson, A. Czarnecka, J. Eriksson, J. Garcia, M. Goniche, C. Hellesen, J. Hobirk, P. Jaquet, E. Joffrin, N. Krawczyk, D. King, M. Lennholm, E. Lerche, E. Pawelec, X. Sáez, M. Sertoli, G. Sips, E. Solano, M. Tsalas, P. Vallejos, M. Valisa, and J. E. T. Contributors. Modelling of JET hybrid plasmas with emphasis on performance of combined ICRF and NBI heating. Nucl. Fusion, 58(10):106037, 2018.

[17] L.-G. Eriksson and M. Schneider. Monte Carlo operators for ions interacting with radio frequency waves. Physics of Plasmas, 12(7):072524, July 2005.

[18] T. Hellsten, T. Johnson, J. Carlsson, L.-G. Eriksson, J. Hedin, M. Laxåback, and M. Mantsinen. Effects of finite drift orbit width and RF-induced spatial transport on plasma heated by ICRH. Nucl. Fusion, 44(8):892, 2004.

[19] R. J. Dumont. Variational approach to radiofrequency waves in magnetic fusion devices. Nucl. Fusion, 49(7):075033, 2009.

[20] Schneider et al. Modelling third harmonic ion cyclotron acceleration of deuterium beams for JET fusion product studies experiments, 2016.

[21] D. Kalupin, I. Ivanova-Stanik, I. Voitsekhovitch, J. Ferreira, D. Coster, L. L. Alves, Th Aniel, J.F Artaud, V. Basiuk, João P. S. Bizarro, R. Coelho, A. Czarnecka, Ph Huynh, A. Figueiredo, J. Garcia, L. Garzotti, F. Imbeaux, F. Köchl, M. F. Nave, G. Pereverzev, O. Sauter, B. D. Scott, R. Stankiewicz, P. Strand, ITM-TF contributors, and JETEFDA Contributors. Numerical analysis of JET discharges with the European Transport Simulator. Nucl. Fusion, 53(12):123007, 2013.

[22] M. Schneider, L.-G. Eriksson, V. Basiuk, and F. Imbeaux. On alpha particle effects in tokamaks with a current hole. Plasma Phys. Control. Fusion, 47(12):2087, 2005.

[23] L.G. Eriksson and P. Helander. Monte Carlo operators for orbit averaged Fokker-Planck equations. Physics of Plasmas, 1(2):308-314, February 1994.

[24] M. Schneider, L.-G. Eriksson, I. Jenkins, J. F. Artaud, V. Basiuk, F. Imbeaux, T. Oikawa, JET-EFDA contributors, and ITM-TF contributors. Simulation of the neutral beam deposition within integrated tokamak modelling frameworks. Nucl. Fusion, 51(6):063019, 2011.

[25] T. Johnson, A. Salmi, G. Steinbrecher, L.-G. Eriksson, T. Hellsten, L. J. Höök, and M. Schneider. Library for RF Interactions in Orbit Following Codes. AIP Conference Proceedings, 1406(1):373-376, December 2011.

[26] Thomas H. Stix. Waves in Plasmas. AIP-Press, 1992. 
[27] Alexei Pankin. The tokamak Monte Carlo fast ion module NUBEAM in the National Transport Code Collaboration library. Computer Physics Communications, (159), 2004.

[28] H.-S. Bosch and G. M. Hale. Improved formulas for fusion cross-sections and thermal reactivities. Nucl. Fusion, 33(12):1919, 1993.

[29] D. R. Mikkelsen. Approximation for non-resonant beam target fusion reactivities. Nucl. Fusion, 29(7):1113, 1989.

[30] J. Garcia, C. Challis, D. Gallart, L. Garzotti, T. Görler, D. King, M. Mantsinen, and J. E. T. contributors. Challenges in the extrapolation from DD to DT plasmas: experimental analysis and theory based predictions for JET-DT. Plasma Phys. Control. Fusion, 59(1):014023, 2017.

[31] Mervi Mantsinen, Jacob Eriksson, Clive Challis, Domenico Frigione, Jerónimo García, Carine Giroud, Ake Hjalmarsson, and King Damian. ICRH analysis of high-performance JET hybrid discharges using PION modelling and neutron spectrometry measurements. volume 39E. European Physical Society (EPS), 2015.

[32] J. F. Artaud, F. Imbeaux, J. Garcia, G. Giruzzi, T. Aniel, V. Basiuk, A. Bécoulet, C. Bourdelle, Y. Buravand, J. Decker, R. Dumont, L. G. Eriksson, X. Garbet, R. Guirlet, G. T. Hoang, P. Huynh, E. Joffrin, X. Litaudon, P. Maget, D. Moreau, R. Nouailletas, B. Pégourié, Y. Peysson, M. Schneider, and J. Urban. Metis: a fast integrated tokamak modelling tool for scenario design. Nucl. Fusion, 58(10):105001, 2018.

[33] J. F. Artaud, V. Basiuk, F. Imbeaux, M. Schneider, J. Garcia, G. Giruzzi, P. Huynh, T. Aniel, F. Albajar, J.M. Ané, A. Bécoulet, C. Bourdelle, A. Casati, L. Colas, J. Decker, R. Dumont, L. G. Eriksson, X. Garbet, R. Guirlet, P. Hertout, G. T. Hoang, W. Houlberg, G. Huysmans, E. Joffrin, S. H. Kim, F. Köchl, J. Lister, X. Litaudon, P. Maget, R. Masset, B. Pégourié, Y. Peysson, P. Thomas, E. Tsitrone, and F. Turco. The CRONOS suite of codes for integrated tokamak modelling. Nucl. Fusion, 50(4):043001, 2010. 